\title{
CENTRAL MASSES AND BROAD-LINE REGION SIZES OF ACTIVE GALACTIC NUCLEI. II. A HOMOGENEOUS ANALYSIS OF A LARGE REVERBERATION-MAPPING DATABASE
}

\author{
B. M. Peterson, ${ }^{1}$ L. Ferrarese, ${ }^{2}$ K. M. Gilbert, ${ }^{1,3}$ S. Kaspi,,${ }^{4}{ }^{5}$ M. A. Malkan, ${ }^{6}$ D. Maoz ${ }^{4}$ D. Merritt ${ }^{7}$ \\ H. Netzer ${ }^{4}$ C. A. Onken, ${ }^{1}$ R. W. Pogge, ${ }^{1}$ M. Vestergatrd, ${ }^{8}$ and A. Wandel ${ }^{9}$ \\ Received 2004 April 5; accepted 2004 June 4
}

\begin{abstract}
We present improved black hole masses for 35 active galactic nuclei (AGNs) based on a complete and consistent reanalysis of broad emission-line reverberation-mapping data. From objects with multiple line measurements, we find that the highest precision measure of the virial product $c \tau \Delta V^{2} / G$, where $\tau$ is the emission-line lag relative to continuum variations and $\Delta V$ is the emission-line width, is obtained by using the cross-correlation function centroid (as opposed to the cross-correlation function peak) for the time delay and the line dispersion (as opposed to FWHM) for the line width and by measuring the line width in the variable part of the spectrum. Accurate line-width measurement depends critically on avoiding contaminating features, in particular the narrow components of the emission lines. We find that the precision (or random component of the error) of reverberationbased black hole mass measurements is typically around 30\%, comparable to the precision attained in measurement of black hole masses in quiescent galaxies by gas or stellar dynamical methods. Based on results presented in a companion paper by Onken et al., we provide a zero-point calibration for the reverberation-based black hole mass scale by using the relationship between black hole mass and host-galaxy bulge velocity dispersion. The scatter around this relationship implies that the typical systematic uncertainties in reverberationbased black hole masses are smaller than a factor of 3 . We present a preliminary version of a mass-luminosity relationship that is much better defined than any previous attempt. Scatter about the mass-luminosity relationship for these AGNs appears to be real and could be correlated with either Eddington ratio or object inclination.
\end{abstract}

Subject headings: galaxies: active — galaxies: nuclei — galaxies: Seyfert — quasars: emission lines

Online material: machine-readable tables

\section{INTRODUCTION}

The evidence that active galactic nuclei (AGNs) are powered by gravitational accretion onto supermassive black holes is now quite convincing. Certainly there has not yet been a definitive detection of the relativistic effects that would be required for unambiguous identification of a singularity, although studies of the $\mathrm{Fe} \mathrm{K} \alpha$ emission line in the X-ray spectra of AGNs currently affords some promise (e.g., Reynolds \& Nowak 2003). Nevertheless it seems to be true that the centers of both active and quiescent galaxies host supermassive (greater than $\sim 10^{6} M_{\odot}$ ) objects that must be so compact that other alternatives are very unlikely.

Black hole masses are measured in a number of ways. In quiescent galaxies, dynamical modeling of either stellar kinematics (e.g., van der Marel 1994, 1998; Verolme et al. 2002;

\footnotetext{
${ }^{1}$ Department of Astronomy, The Ohio State University, 140 West 18th Avenue, Columbus, OH 43210; peterson@astronomy.ohio-state.edu, kgilbert@astro.ucsc .edu, onken@astronomy.ohio-state.edu, pogge@astronomy.ohio-state.edu.

${ }^{2}$ Department of Physics and Astronomy, Rutgers University, 136 Frelinghuysen Road, Piscataway, NJ 08854; 1ff@physics.rutgers.edu.

${ }^{3}$ Current address: University of California Observatories, Lick Observatory, University of California, Santa Cruz, CA 92064.

${ }^{4}$ Wise Observatory and School of Physics and Astronomy, Raymond and Beverly Sackler Faculty of Exact Sciences, Tel Aviv University, Tel Aviv 69978, Israel; shai@wise.tau.ac.il, dani@wise.tau.ac.il; netzer@wise.tau.ac.il.

5 Department of Physics, Technion, Haifa 32000, Israel.

6 Department of Physics and Astronomy, University of California, Los Angeles, CA 90095; malkan@bonnie.astro.ucla.edu.

7 Department of Physics, Rochester Institute of Technology, 84 Lomb Memorial Drive, Rochester, NY 14623; drmsps@ad.rit.edu.

${ }^{8}$ Steward Observatory, University of Arizona, Tucson, AZ 85721, mvestergaard@as.arizona.edu.

${ }_{9}$ Racah Institute of Physics, The Hebrew University, Jerusalem 91405, Israel; amri@vms.huij.ac.il.
}

Gebhardt et al. 2003) or gas motions (e.g., Harms et al. 1994; Ford et al. 1994; Macchetto et al. 1997) is used to determine central masses. In the case of NGC 4258, a weakly active galaxy, proper motions and radial velocities of $\mathrm{H}_{2} \mathrm{O}$ megamaser spots are used to deduce a high precision central mass (Miyoshi et al. 1995; Herrnstein et al. 1999). In Type 1 active galaxies (i.e., those with prominent broad emission lines in their UV/ optical spectra), reverberation mapping (Blandford \& McKee 1982; Peterson 1993) of the broad-line region (BLR) can be used to determine the central masses. Reverberation mapping is the only method that does not depend on high angular resolution, so it is of special interest since it is thus extendable in principle to both very high and very low luminosities and to objects at great distances. Moreover, reverberation studies reveal the existence of simple scaling relationships that can be used to anchor secondary methods of mass measurement, thus making it possible to provide estimates of the masses of large samples of quasars, including even very distant quasars, based on relatively simple spectral measurements (e.g., Vestergaard 2002, 2004; McLure \& Jarvis 2002).

While reverberation methods in principle can be used to determine the full geometry and kinematics of the BLR (e.g., Horne et al. 2004), applications to date have been comparatively simple. Time delays between continuum and emission-line variations are used to deduce the size of the BLR, or more accurately, the size of the line-emitting region for the particular emission line in question. By combining the measured time delay $\tau$ with the emissionline width $\Delta V$, a virial mass can be obtained,

$$
M=\frac{f_{c} \tau \Delta V^{2}}{G}
$$


where $c$ is the speed of light and $G$ is the gravitational constant. The factor $f$ is of order unity and depends on the structure, kinematics, and orientation of the BLR. We will sometimes refer below to the "unscaled" virial mass $M_{p}=c \tau \Delta V^{2} / G$, as the virial product, so that the virial mass is the virial product times the scaling factor $f$.

It became clear even in the first well-sampled reverberation program on NGC 5548 (Clavel et al. 1991; Peterson et al. 1991; Dietrich et al. 1993; Maoz et al. 1993) that different emission lines have different time-delayed responses, or lags. Lags are shorter for lines that are characteristic of more highly ionized gases, i.e., the BLR has a stratified ionization structure. It was already known (e.g., Osterbrock \& Shuder 1982) that higher ionization lines (e.g., He II 24686 ) are broader than lower ionization lines (e.g., $\mathrm{H} \beta$ 24861), and it was natural to look for a virial relationship between lag and line width, $\tau \propto \Delta V^{-2}$, which would constitute evidence that gravity dominates the motions of the BLR gas and that the black hole mass can therefore be inferred. Early attempts to do this were not promising, although Krolik et al. (1991) did note the trend of decreasing time lag with increasing line width for the UV lines in NGC 5548. However, upon revisiting the issue, Peterson \& Wandel (1999) found that there is indeed a virial relationship between lag and line width in the case of NGC 5548 if the line width is measured in the variable part of the emission line and one avoids (1) lines that are strongly blended with other features and (2) lines with lags that are uncertain because of potential aliasing effects in the time-series analysis. Similar virial-like relationships between lag and line width were subsequently found in other objects (Peterson \& Wandel 2000; Onken \& Peterson 2002; Kollatschny 2003). Despite earlier claims that emission-line reverberation yielded masses that were too low by a factor of several (Ho 1999), it was subsequently shown (Gebhardt et al. 2000b; Ferrarese et al. 2001) that the relationship between AGN reverberation-based black hole masses $M_{\mathrm{BH}}$ and their host-galaxy bulge velocity dispersions $\sigma_{*}$ appears to be consistent with the black hole mass/bulge velocity dispersion relationship (hereafter the $M_{\mathrm{BH}^{-}} \sigma_{*}$ relationship; Ferrarese \& Merritt 2000; Gebhardt et al. 2000a) that is seen in normal galaxies. Moreover, the relationship between black hole mass and host-galaxy bulge luminosity also seems to be the same for both quiescent and active galaxies (Wandel 2002; McLure \& Dunlop 2002).

Unfortunately, there is a significant systematic uncertainty (Krolik 2001) in AGN reverberation masses embodied in the scaling factor $f$ in equation (1), which remains unknown. For lack of a better estimate, published studies have usually used a nominal value of $f=0.75$ for $\Delta V$ taken to be the FWHM of the emission line, as described in $\S 6$ below.

Wandel et al. (1999; hereafter Paper I) published a compilation of black hole masses in 17 Seyfert 1 galaxies and two quasars. Kaspi et al. (2000) published the results of a large reverberation-mapping campaign that led to mass measurements for the central objects in 17 Palomar-Green (Schmidt \& Green 1983) quasars and combined their results with Paper I to obtain relationships between the BLR size and AGN optical luminosity (the "radius-luminosity relationship") and between the central mass, which we will henceforth assume to be a black hole, and the optical luminosity (the "mass-luminosity relationship"). These are both of obvious importance:

1. The radius-luminosity relationship can be used to deduce the masses of black holes in distant quasars by combining the inferred BLR radius with the widths of the emission lines.
2. The mass-luminosity relationship hence obtained relates directly to current accretion rates and radiative efficiencies. The mass itself provides a strong constraint on the black hole growth history.

All of the important relationships mentioned here-the time-lag/line-width virial relationship, the AGN $M_{\mathrm{BH}^{-}} \sigma_{*}$ relationship, the radius-luminosity relationship, and the massluminosity relationship - show considerable scatter. Moreover, the reverberation database is very inhomogeneous, and the data have not always been analyzed in a uniform way; this is particularly true in the case of many of the earliest results. We suspected that more than a decade of experience in developing reverberation mapping techniques and error analysis merited reanalysis of the earlier data, and that in at least some cases, improved calibration for spectra would lead to improved results. This suspicion was borne out in the case of NGC 3783 (Onken \& Peterson 2002); our reanalysis of the combined UV and optical data led to a determination of the virial mass of the central object that was an order of magnitude more precise than that quoted by Paper I, which was based only on the original optical spectra and optical continuum and $\mathrm{H} \beta$ light curves. Fundamentally, a complete reanalysis of the body of reverberation database is warranted by relatively recent (1) improvements in cross-correlation error analysis, (2) recognition of the importance of measuring line widths in the variable part of the spectrum, and (3) recognition that emission-line time lags can vary over timescales longer than the reverberation timescale due to changes in the mean luminosity of the object (Peterson et al. 2002).

We thus decided to undertake a massive reanalysis of all of the reverberation mapping data readily available to us, for the express purpose of improving AGN black hole mass determinations. We distinguish in the usual way (e.g., Bevington 1969) between the accuracy of these masses (i.e., how close they are to the true values), which depends on how well we can account for systematics, and their precision (i.e., how exactly we measure the virial products), which depends primarily on "random errors" associated with measuring line widths and time lags. Thus, this investigation has two parts, with different goals:

1. In order to improve the precision of AGN black hole mass measurements, we are reanalyzing all of the readily available reverberation data to determine the best measures of time lag and line width for these studies. We do this by assuming that the virial product for all emission lines is the same at all times for a particular AGN. We then explore ways of characterizing lags and line widths that yield relationships most consistent with a virial relationship. This is the subject of this contribution.

2. In order to improve the accuracy of AGN black hole mass measurements, we are obtaining high-precision measurements of $\sigma_{*}$ for reverberation-mapped AGNs. We demonstrate that there is general consistency between the $M_{\mathrm{BH}^{-}} \sigma_{*}$ relationships between AGNs and quiescent galaxies, and then assume that these two relationships have a common zero point, thus determining the scale factor $f$. This is the subject of a companion paper (Onken et al. 2004), which we will draw on for the absolute calibration of the black hole mass scale.

\section{DATA}

We have included in this analysis all objects for which we had ready access to the spectra used in the original investigations. This consists of data from most of the reverberationmapping experiments undertaken to date, including the large 
TABLE 1

ОвJECT LIST

\begin{tabular}{|c|c|c|c|c|c|c|c|c|}
\hline $\begin{array}{l}\text { Objects } \\
\text { (1) }\end{array}$ & $\begin{array}{c}\text { References }^{\mathrm{a}} \\
\text { (2) }\end{array}$ & $\begin{array}{c}\alpha \\
(\mathrm{J} 2000.0) \\
(3)\end{array}$ & $\begin{array}{c}\delta \\
(\mathrm{J} 2000.0) \\
(4)\end{array}$ & $\begin{array}{c}z \\
(5)\end{array}$ & $\begin{array}{c}V \\
(\mathrm{mag}) \\
(6)\end{array}$ & $\begin{array}{c}A_{B} \\
(\mathrm{mag}) \\
(7)\end{array}$ & $\begin{array}{c}\text { Véron-Cetty \& } \\
\text { Véron Catalog Name } \\
\text { (8) }\end{array}$ & $\begin{array}{c}\text { Alternative Name } \\
\text { (9) }\end{array}$ \\
\hline PG $0026+129 \ldots \ldots$. & 2 & 002913.600 & +131603.00 & 0.14200 & 15.4 & 0.307 & J002913.8+131605 & \\
\hline PG $0052+251 \ldots \ldots$. & 2 & 005452.100 & +252538.00 & 0.15500 & 15.4 & 0.205 & $\mathrm{~J} 005452.2+252539$ & \\
\hline Fairall $9 \ldots \ldots \ldots \ldots \ldots$ & 3,4 & 012345.780 & -584820.50 & 0.04702 & 13.5 & 0.116 & J012345.8-584821 & \\
\hline Akn $120 \ldots \ldots \ldots \ldots \ldots$ & 1 & $05 \quad 1611.421$ & -000859.38 & 0.03230 & 14.1 & 0.554 & J051611.4-000900 & Mrk 1095 \\
\hline Mrk 79 .................. & 1 & 074232.797 & +494834.75 & 0.02219 & 13.9 & 0.305 & J074232.8+494835 & \\
\hline PG $0804+761 \ldots \ldots$. & 2 & 081058.600 & +760242.00 & 0.10000 & 15.1 & 0.150 & J081058.5+760243 & \\
\hline PG $0844+349 \ldots \ldots$. & 2 & 084742.400 & +344504.00 & 0.06400 & 14.0 & 0.159 & J084742.5+344505 & \\
\hline 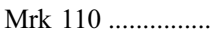 & 1 & 092512.870 & +52 1710.52 & 0.03529 & 16.0 & 0.056 & J092512.9+521711 & \\
\hline PG $0953+414 \ldots \ldots$. & 2 & 095652.400 & +411522.00 & 0.23410 & 14.5 & 0.054 & J095652.3+411522 & \\
\hline NGC $3227 \ldots \ldots \ldots \ldots . . . .$. & $5,6,7$ & 102330.589 & +195153.99 & 0.00386 & 11.1 & 0.098 & $\mathrm{~J} 102330.6+195156$ & \\
\hline PG $1211+143 \ldots \ldots$. & 2 & 121417.700 & +140312.60 & 0.08090 & 14.6 & 0.150 & $\mathrm{~J} 121417.7+140313$ & \\
\hline PG $1226+023 \ldots \ldots$. & 2 & 122906.700 & +020308.60 & 0.15834 & 12.8 & 0.089 & $\mathrm{~J} 122906.7+020308$ & $3 \mathrm{C} 273$ \\
\hline PG $1229+204 \ldots \ldots$. & 2 & 123203.605 & +200929.21 & 0.06301 & 15.3 & 0.117 & $\mathrm{~J} 123203.6+200930$ & Mrk 771, Ton 1542 \\
\hline NGC $4593 \ldots \ldots \ldots . . .$. & 6,15 & 123939.425 & -052039.34 & 0.00900 & 11.7 & 0.106 & J123939.4-052039 & Mrk 1330 \\
\hline PG $1307+085 \ldots \ldots$. & 2 & 130947.000 & +08 1948.90 & 0.15500 & 15.3 & 0.145 & J130947.0+081949 & \\
\hline 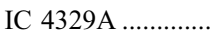 & 16 & 134919.29 & -301834.4 & 0.01605 & 14.0 & 0.255 & J134919.3-301834 & \\
\hline Mrk 279 & 17,18 & 135303.447 & +69 18 29.57 & 0.03045 & 14.6 & 0.068 & $\mathrm{~J} 135303.5+691830$ & \\
\hline PG $1351+640 \ldots \ldots$. & 2 & 135315.808 & +634545.41 & 0.08820 & 14.8 & 0.088 & $\mathrm{~J} 135315.7+634546$ & \\
\hline PG $1411+442 \ldots \ldots$. & 2 & 141348.300 & +440014.00 & 0.08960 & 15.0 & 0.036 & $\mathrm{~J} 141348.3+440014$ & \\
\hline NGC 5548................ & $19,20,21,22,23$ & 141759.534 & +2508 12.44 & 0.01717 & 13.3 & 0.088 & $\mathrm{~J} 141759.6+250813$ & \\
\hline PG $1426+015 \ldots \ldots$. & 2 & 142906.588 & +011706.48 & 0.08647 & 17.5 & 0.137 & $\mathrm{~J} 142906.5+011704$ & Mrk 1383 \\
\hline 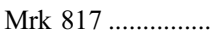 & 1 & 143622.068 & +584739.38 & 0.03145 & 14.5 & 0.029 & $\mathrm{~J} 143622.1+584740$ & PG $1434+590$ \\
\hline PG $1613+658 \ldots \ldots .$. & 2 & 161357.179 & +654309.58 & 0.12900 & 15.2 & 0.114 & $\mathrm{~J} 161357.2+654309$ & Mrk 876 \\
\hline
\end{tabular}

NoтE.-Units of right ascension are hours, minutes, and seconds, and units of declination are degrees, arcminutes, and arcseconds.

${ }^{a}$ References: (1) Peterson et al. 1998a; (2) Kaspi et al. 2000; (3) Santos-Lleó et al. 1997; (4) Rodríguez-Pascual et al. 1997; (5) Salamanca et al. 1994; (6) Onken et al. 2003; (7) Winge et al. 1995; (8) Wanders et al. 1993; (9) Stirpe et al. 1994; (10) Onken \& Peterson 2002; (11) Reichert et al. 1994; (12) Peterson et al. 2000; (13) Kaspi et al. 1996; (14) Maoz et al. 1991; (15) Dietrich et al. 1994; (16) Winge et al. 1996; (17) Santos-Lleó et al. 2001; (18) Maoz et al. 1990; (19) Peterson et al. 2002 and references therein; (20) Dietrich et al. 1993; (21) Clavel et al. 1991; (22) Korista et al. 1995; (23) Netzer et al. 1990; (24) Dietrich et al. 1998; (25) O'Brien et al. 1998; (26) Collier et al. 1998; (27) Wanders et al. 1997.

samples from International AGN Watch ${ }^{10}$ projects (Alloin et al. 1994; Peterson 1999), the Lovers of Active Galaxies (LAG) campaign (Robinson 1994), the Ohio State monitoring program (Peterson et al. 1998a), and the Wise Observatory/ Steward Observatory monitoring program (Kaspi et al. 2000). A list of objects analyzed here is given in Table 1. Column (1) gives the common name of the object as used in the relevant papers on the reverberation results, which are referenced in column (2). Epoch 2000 coordinates are given in columns (3) and (4). The redshift $z$ of each object is in column (5), with nominal $V$ magnitude and $B$-band extinction, based on $100 \mu \mathrm{m}$ dust maps from Schlegel et al. (1998), are given in columns (6)

\footnotetext{
${ }^{10}$ Data obtained as part of International AGN Watch projects are available at http://www.astronomy.ohio-state.edu/ agnwatch.
}

and (7), respectively. Column (8) gives the standard name of the object in the Véron-Cetty \& Véron (2001) catalog, and column (9) gives other common names by which the object is often known in the AGN literature. All entries in this table are from the NASA/IPAC Extragalactic Database.

For this analysis, the fundamental data that we require are (1) continuum and emission-line light curves to determine time lags and (2) spectra from which line widths are to be measured. We use the published versions of the light curves, except where noted below. For the purpose of measuring the line widths, we use the same spectra from which the continuum and emissionline measurements were made; in some cases, notably the groundbased component of the International AGN Watch monitoring programs on Fairall 9, NGC 3783, NGC 4051, NGC 4151, NGC 5548, 3C 390.3, and NGC 7469, we restricted consideration to the single largest homogeneous data subsets, i.e., those 
TABLE 2

Wavelength Windows for Line Width Measurements

\begin{tabular}{|c|c|c|c|c|c|c|c|}
\hline $\begin{array}{l}\text { Object } \\
\text { (1) }\end{array}$ & $\begin{array}{c}\text { Reference }^{\mathrm{a}} \\
\text { (2) }\end{array}$ & $\begin{array}{c}\text { Data Set } \\
\text { (3) }\end{array}$ & $\begin{array}{c}\text { Resolution } \\
(\AA) \\
(4)\end{array}$ & $\begin{array}{c}\text { Julian Dates } \\
(-2,400,000) \\
(5)\end{array}$ & $\begin{array}{c}\text { Continuum Window } \\
(\AA) \\
(6)\end{array}$ & $\begin{array}{l}\text { Continuum Window } \\
\qquad \begin{array}{c}(\AA) \\
(7)\end{array}\end{array}$ & $\begin{array}{c}\text { Line Limits } \\
(\AA) \\
(8)\end{array}$ \\
\hline & 1 & $\mathrm{H} \beta$ & 9.6 & $49,889-50,118$ & $4910-4920$ & $5100-5110$ & $4930-5030$ \\
\hline \multirow[t]{2}{*}{ PG $0026+129 \ldots \ldots \ldots$} & 2 & $\mathrm{H} \alpha$ & 12.5 & $48,836-51,084$ & $7290-7300$ & $7700-7740$ & $7304-7728$ \\
\hline & 2 & $\mathrm{H} \beta$ & 12.5 & $48,545-51,084$ & $5442-5450$ & $5820-5840$ & $5460-5700$ \\
\hline PG $0052+251 \ldots \ldots \ldots$ & 2 & $\mathrm{H} \alpha$ & 12.5 & $48,837-51,084$ & $7028-7108$ & $7725-7800$ & $7398-7724$ \\
\hline & 2 & $\mathrm{H} \gamma$ & 12.5 & $48,461-51,084$ & 4864-4914 & $5418-5488$ & $4926-5118$ \\
\hline \multirow[t]{3}{*}{ 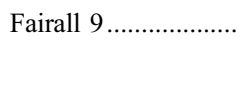 } & 3 & $\mathrm{H} \beta$ & 11.2 & $50,473-50,665$ & $4936-4946$ & $5295-5323$ & $4946-5295$ \\
\hline & 4 & C IV $\lambda 1549$ & 6.0 & $50,473-50,713$ & $1540-1565$ & $1760-1770$ & $1565-1670$ \\
\hline & 4 & $\operatorname{Ly} \alpha$ & 6.0 & $50,473-50,713$ & $1227-1232$ & $1370-1380$ & $1232-1334$ \\
\hline
\end{tabular}

NoтE.-Table 2 is also available in machine-readable form in the electronic edition of the Astrophysical Journal.

${ }^{a}$ References are as in Table 1.

data that are most similar in terms of resolution and quality, often from a single source. For each set of spectra, we formed a mean spectrum,

$$
\overline{F(\lambda)}=\frac{1}{N} \sum_{i=1}^{N} F_{i}(\lambda)
$$

where $F_{i}(\lambda)$ is the $i$ th spectrum of the $N$ spectra that compose the database. We also define an rms spectrum:

$$
S(\lambda)=\left\{\frac{1}{N-1} \sum_{i=1}^{N}\left[F_{i}(\lambda)-\overline{F(\lambda)}\right]^{2}\right\}^{1 / 2} .
$$

We form the mean and rms spectra only from the most homogeneous subsets of the database, taking care to ensure that variations observed in the homogeneous subset are consistent with those observed in the entire data set. Strict homogeneity of the data, particularly in terms of spectral resolution and spectrograph entrance aperture, is necessary to avoid introduction of spurious features in the mean and rms spectra.

Line widths can be measured in either the mean or rms spectrum; the advantage of using the rms spectrum is that constant components of the spectrum, or those that vary on timescales much longer than the duration of the experiment, vanish, thus largely obviating the problem of deblending lines. The corresponding disadvantage, however, is that the rms spectra are generally much noisier than the mean spectra as the amplitude of variability is usually fairly small for these AGNs. The most compelling reason to use the rms spectra is that then we are measuring the parts of the emission lines that are actually varying. We thus have a strong prejudice toward using the rms spectra and attempt to justify this choice below.

For several of the galaxies listed in Table 1, there are multiple data sets available, sometimes from the same source and sometimes from different sources. We analyze each individual set as an independent time series. In the case of some of the brighter Seyfert 1 galaxies that have relatively short $\mathrm{H} \beta$ response times, multiple-year campaigns were broken down into individual subsets covering single observing seasons, thus yielding multiple, independent measurements of the line widths, lags, and virial masses. This is desirable not only from a statistical point of view, but because it is now clear (Peterson et al. 2002) that both lags and line widths can vary from one observing season to the next as the mean continuum luminosity slowly varies.

\section{LINE WIDTHS}

In Table 2 we identify each individual data set for which time-series analysis was carried out. Column (1) gives the common name of the object, and column (2) gives the reference for the original data. Individual emission lines are identified in column (3) and the spectral resolution of the data (see below) appears in column (4). Column (5) gives the range in Julian dates spanned by the spectra. ${ }^{11}$ Individual emission lines were isolated by interpolating a linear continuum (in units of ergs $\mathrm{s}^{-1} \mathrm{~cm}^{-2} \AA^{-1}$ ) between continuum windows on either side of the line (cols. [6] and [7]) underneath the line, whose limits are given in column (8); all wavelengths in Table 2 are in the observer's reference frame.

\subsection{Measures of Line Width}

Given an emission-line profile $P(\lambda)$ (i.e., flux per unit wavelength above a continuum interpolated underneath the line), we parameterized the line width in two separate ways:

FWHM.-How this quantity is measured depends on whether the line is single- or double-peaked. In the case of a single-peaked line, we identify the line peak $P(\lambda)_{\max }$. We then start at the short-wavelength limit of the line (col. [8] of Table 2) and search for the $\lambda_{1}$ such that $P\left(\lambda_{1}\right)=0.5 P(\lambda)_{\max }$. We then repeat the search starting from the line peak and moving to shorter wavelengths to find $\lambda_{2}$ such that $P\left(\lambda_{2}\right)=0.5 P(\lambda)_{\max }$. The mean of these two wavelengths is taken to be the wavelength at half-maximum flux on the short-wavelength side of the profile. An identical procedure is used to identify the half-maximum point on the long-wavelength side of the line, and the difference between these is taken to be the FWHM. For a double-peaked line, we define a short-wavelength peak $P(\lambda)_{\max , \text { short }}$ and a long-wavelength peak $P(\lambda)_{\max , \text { long }}$. We then follow procedures similar to those above: we define $\lambda_{1}$ and $\lambda_{2}$ relative to the short-wavelength peak only and compute their mean. A similar calculation is done on the long-wavelength side, this time relative to $P(\lambda)_{\max }$ long, and the FWHM is taken

\footnotetext{
${ }^{11}$ It should be noted that in many cases, isolated points at the beginning or end of the original time series may be excluded from our analysis.
} 


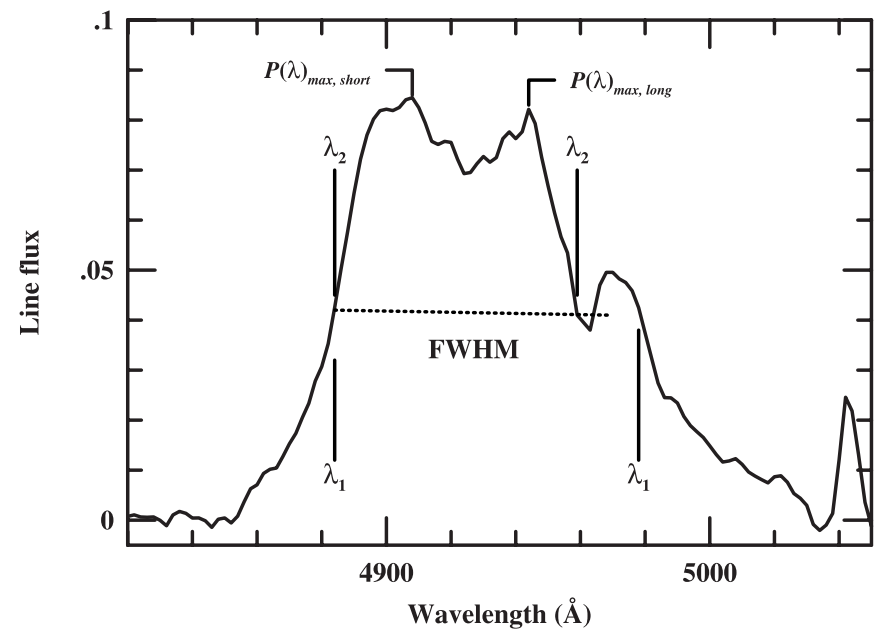

FIG. 1.-Measurement of FWHM for double-peaked profiles. A continuum is interpolated underneath the line profile and is subtracted from the data. Peak fluxes are identified on the short-wavelength and long-wavelength peaks, $P(\lambda)_{\max , \text { short }}$ and $P(\lambda)_{\max }$ long, respectively. On each side of the line, wavelengths corresponding to the half-maximum fluxes $0.5 P(\lambda)_{\max \text {, short }}$ and $0.5 P(\lambda)_{\max }$ long are found moving upward from the continuum at $\lambda_{1}$ and downward from the peak at $\lambda_{2}$. In this example, $\lambda_{1}=\lambda_{2}$ on the short-wavelength side only. The nominal position of the half-maximum point is taken to be the average of $\lambda_{1}$ and $\lambda_{2}$.

to be the separation between the calculated means of $\lambda_{1}$ and $\lambda_{2}$ on either side of the line. This is illustrated in Figure 1.

Line dispersion.- The first moment of the line profile is

$$
\lambda_{0}=\int \lambda P(\lambda) d \lambda / \int P(\lambda) d \lambda
$$

We use the second moment of the profile to define the variance or mean square dispersion

$$
\sigma_{\text {line }}^{2}(\lambda)=\left\langle\lambda^{2}\right\rangle-\lambda_{0}^{2}=\left[\int \lambda^{2} P(\lambda) d \lambda / \int P(\lambda) d \lambda\right]-\lambda_{0}^{2} .
$$

The square root of this equation is the line dispersion $\sigma_{\text {line }}$ or rms width of the line.

Both measures have intrinsic strengths and weaknesses: FWHM is trivial to measure, except in the case of multiplepeaked lines or noisy data, and can even be accurately estimated graphically. Compared to $\sigma_{\text {line }}$, it is also less sensitive to blending with other lines and to the contribution from extended line wings. On the other hand, $\sigma_{\text {line }}$ is well defined for arbitrary line profiles, less sensitive to the presence of even fairly strong narrow-line components, and as we shall see throughout this analysis, more accurate for low-contrast lines, and the relative uncertainties are much lower than for FWHM. On the other hand, $\sigma_{\text {line }}$ is in some cases also problematic; $\sigma_{\text {line }} \rightarrow \infty$ for a Lorentzian profile, for example (although in practice, the wings of any reasonable line profile become lost in the noise). Fromerth \& Melia (2000) point out many of the advantages of $\sigma_{\text {line }}$ relative to FWHM and show that a virial relationship between lag and line width is also found using $\sigma_{\text {line }}$ rather than FWHM to characterize the line width.

It is worth noting at this point that there is a simple relationship between these two quantities for a given line profile. For a Gaussian, FWHM $/ \sigma_{\text {line }}=2(2 \ln 2)^{1 / 2} \approx 2.355$, and for a rectangular profile (produced by emission-line clouds in circular Keplerian orbits of fixed radius and random inclination), $\mathrm{FWHM} / \sigma_{\text {line }}=2(3)^{1 / 2} \approx 3.464$.
TABLE 3

\begin{tabular}{|c|c|}
\hline Object & $\begin{array}{c}\text { FWHM ([O III] }] \text { 25007) } \\
\left(\mathrm{km} \mathrm{s}^{-1}\right)\end{array}$ \\
\hline Mrk $335 \ldots$. & 280 \\
\hline Fairall $9 \ldots \ldots \ldots$ & 425 \\
\hline Mrk $590 \ldots \ldots \ldots . . .$. & 400 \\
\hline Akn 120 & 490 \\
\hline 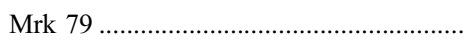 & 350 \\
\hline 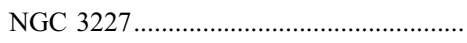 & 485 \\
\hline NGC 3516 & 250 \\
\hline NGC $3783 \ldots \ldots$ & 230 \\
\hline 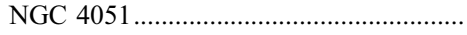 & 190 \\
\hline 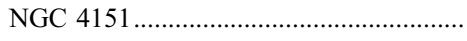 & 425 \\
\hline 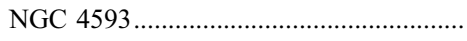 & 255 \\
\hline IC 4329A & 550 \\
\hline 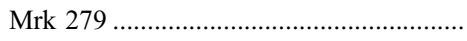 & 580 \\
\hline 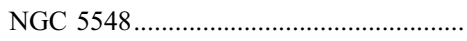 & 410 \\
\hline 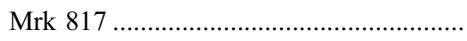 & 330 \\
\hline Mrk 509 & 520 \\
\hline NGC 7469 & 360 \\
\hline
\end{tabular}

[O III] $\lambda 5007$ LiNE WidTHS

\subsection{Resolution Correction}

Since some of the emission lines widths are actually rather narrow, we need to correct each line-width measurement for the finite resolution of the spectrograph with which the data were obtained. We will assume that the observed line widths $\Delta \lambda_{\text {obs }}$ can be written in terms of the intrinsic line widths $\Delta \lambda_{\text {true }}$ and the spectrograph resolution $\Delta \lambda_{\text {res }}$ as

$$
\Delta \lambda_{\mathrm{obs}}^{2} \approx \Delta \lambda_{\text {true }}^{2}+\Delta \lambda_{\text {res }}^{2}
$$

Application of this equation to obtain $\Delta \lambda_{\text {true }}$ requires knowing the resolution at which the observations were made. In order to determine this for the optical data used here, we relied on accurate, high-resolution measurements of the width of the [O III] 25007 line in many of the AGNs discussed here by Whittle (1992). Whittle's FWHM measurements for AGNs in this study are given in Table 3 , in the rest frame of each galaxy. In order to determine the resolution of the data used in this study, we transformed the values in Table 3 back to the observed frame and to wavelengths units and assumed this to be $\Delta \lambda_{\text {true }}$. We then took our measurements of FWHM([O III $] 25007)$ as $\Delta \lambda_{\text {obs }}$ and solved for $\Delta \lambda_{\text {res }}$, the FWHM resolution of the data. These are the values given in column (4) of Table 2 . We recover $\Delta \lambda_{\text {true }}$ for the broad lines by application of equation (6).

The absence of isolated narrow lines in the UV spectra of AGNs precluded using this method for UV spectra. Instead, we assume a spectral resolution of $6 \AA$ for the International Ultraviolet Explorer (IUE) SWP camera and a resolution of $1.9 \AA$ for Hubble Space Telescope (HST) Faint Object Spectrograph spectra (e.g., Korista et al. 1995).

\subsection{Narrow-Line Contamination}

As noted above, in principle constant line components should not appear in rms spectra. In practice, we find that residual narrow-line features, generally weak but sometimes quite strong, appear in our rms spectra. These narrow-line residuals appear when the data are less than ideally homogeneous, in particular, when the line-spread function is not the same for each spectrum. The Wise Observatory spectra are particularly prone to this because of the method used to effect a photometric calibration of the spectra; these data are obtained 
TABLE 4

Strength of Narrow-H $\beta$ Component

\begin{tabular}{|c|c|c|}
\hline Object & $F\left(\mathrm{H} \beta_{\text {narrow }}\right) / F\left(\left[\begin{array}{ll}\text { III }] & \lambda 5007)\end{array}\right.\right.$ & References \\
\hline PG $0026+129 \ldots \ldots \ldots \ldots \ldots \ldots$ & 0.108 & 1 \\
\hline PG $0052+251 \ldots \ldots \ldots \ldots \ldots \ldots$ & 0.120 & 1 \\
\hline 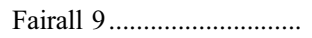 & 0.121 & 1 \\
\hline PG $0953+414 \ldots \ldots \ldots \ldots \ldots \ldots$ & 0.147 & 1 \\
\hline 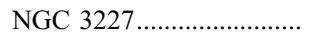 & 0.088 & 2 \\
\hline 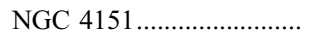 & 0.084 & 2 \\
\hline PG $1229+204 \ldots \ldots \ldots \ldots \ldots \ldots$ & 0.080 & 2 \\
\hline 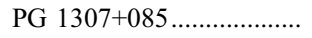 & 0.094 & 1 \\
\hline 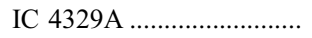 & 0.136 & 1 \\
\hline 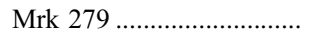 & 0.215 & 1 \\
\hline PG $1351+640 \ldots \ldots \ldots \ldots \ldots \ldots$ & 0.330 & 1 \\
\hline 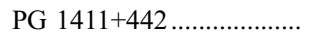 & 0.213 & 1 \\
\hline NGC $5548 \ldots \ldots \ldots \ldots \ldots \ldots \ldots \ldots$ & 0.110 & 2 \\
\hline PG $1426+015 \ldots \ldots \ldots \ldots \ldots \ldots$ & 0.120 & 2 \\
\hline PG $1613+658 \ldots \ldots \ldots \ldots \ldots \ldots$ & 0.075 & 1 \\
\hline PG $1617+175 \ldots \ldots \ldots \ldots \ldots \ldots$ & 0.192 & 1 \\
\hline PG $1704+608 \ldots \ldots \ldots \ldots \ldots \ldots$ & 0.110 & 2 \\
\hline 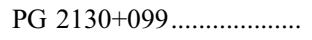 & 0.306 & 1 \\
\hline NGC $7469 \ldots \ldots \ldots \ldots \ldots \ldots \ldots$ & 0.181 & 1 \\
\hline
\end{tabular}

References.-(1) Marziani et al. 2003; 2: This work.

through a long, wide slit that also contains a nearby field star that is used for relative photometric calibration. An unfortunate side effect of this highly accurate photometric calibration method is that the line-spread function is not well-controlled as the target can migrate small amounts within the slit in the dispersion direction; while this has only a small effect on the measured broad-line widths, the narrow-line profiles are strongly affected and fairly strong narrow-line residuals can result in the rms spectrum. Again as we will see below, accurate narrow-line removal is critical for accurate line-width measurement. Therefore, in cases where the rms spectra show significant residual narrow components of $\mathrm{H} \beta$ and/or [O III] $\lambda \lambda 4959,5007$, we have subtracted these components from each spectrum before combining them into mean and rms spectra. In such cases, we used [O III 25007 as a template profile and shifted and flux-scaled this profile to obtain a suitable model of the narrow lines, which we then subtracted from each spectrum prior to forming the mean and rms spectra. The $[\mathrm{O}$ III] $\lambda 5007 /[\mathrm{O}$ III] $\lambda 4959$ flux ratio is fixed at a value $\sim 3$ by atomic physics, but the $\mathrm{H} \beta_{\text {narrow }} /[\mathrm{O}$ III] $\lambda 5007$ flux ratio is different for each galaxy. For galaxies in which narrow-line removal was undertaken for even some of the data, the adopted narrow-line fluxes are shown in Table 4. Most of these values are from Marziani et al. (2003), although in few cases we used our own determinations.

Unfortunately, decomposition of the narrow lines from the spectra is much more difficult in the $\mathrm{H} \alpha$ and $\mathrm{H} \gamma$ regions of the optical spectrum and was therefore not attempted. Cases in which this might present a problem are noted below. Finally, we note that none of the UV data from IUE or HST show narrowline residuals, consistent with little or no narrow-line contribution to the UV emission lines in quasars (see Wills et al. 1993).

\subsection{Line Width Uncertainties}

To determine FWHM and $\sigma_{\text {line }}$ and their associated uncertainties, we employ a bootstrap method similar to that which we use for the time-series analysis. A given data set contains $N$ spectra. For a single bootstrap realization, we make $N$ random selections from this group, without regard to whether or not a

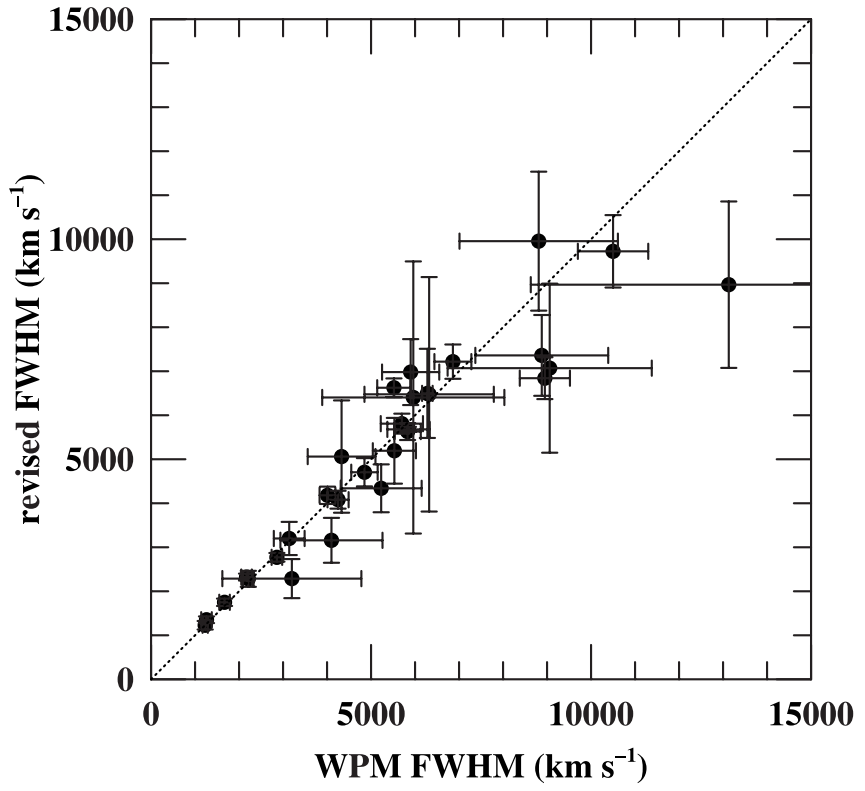

FIG. 2.-Comparison of FWHM measurements and uncertainties from this paper with those from Paper I. For the sake of meaningful comparison, the new measurements here have not been corrected for spectral resolution, as have all other line-width measurements in this paper.

particular spectrum has been previously selected. From these $N$ spectra we form a mean spectrum and an rms spectrum (eqs. [2] and [3]), and fits to the underlying continuum and the line measurements are performed on these spectra. Multiple bootstrap realizations allow us to build up a distribution of linewidth measurements from the random sets of $N$ spectra. From these we can compute a mean value and standard deviation for the width of each emission line, and these are the values that we will use in this analysis.

This method of determining the line widths and associated errors is different than what we have done previously. Line widths and uncertainties presented by Paper I were determined less rigorously, by comparing the measurements of FWHM obtained by using the "highest plausible" and "lowest plausible" underlying continua. Figure 2 shows a comparison between FWHM values and associated uncertainties from this study and those reported by Paper I; note that for this particular comparison only, we did not adjust our measurements for spectral resolution in order to effect a more meaningful comparison with Paper I. In general, the measurements and errors are both in good agreement. The uncertainties we find here are on average about $12 \%$ lower than those quoted by Paper I.

The results on two quasars in Table 1, PG $1351+640$ and PG $1704+608$, were deemed to be too poor to retain in this analysis; the emission-line variability was simply too weak to produce reliably measurable emission lines in the rms spectra. We note in particular that the problem with PG 1704+608 has already been discussed in the literature (Boroson 2003); the $\mathrm{H} \beta$ line in the rms spectrum is not in fact variable broad-line emission, but merely residual narrow-line emission.

\section{TIME SERIES ANALYSIS}

The methodology we employ for measurement of time lags and their associated errors is the interpolation cross-correlation method, essentially as described by White \& Peterson (1994) and by Peterson et al. (1998b, hereafter P98b), but with some modifications that are described in the Appendix. A complete tutorial on our cross-correlation methodology is provided by 
TABLE 5

Time-Series Analysis

\begin{tabular}{|c|c|c|c|c|c|c|c|}
\hline \multirow[b]{2}{*}{$\begin{array}{l}\text { ОвЈест } \\
\text { (1) }\end{array}$} & \multirow[b]{2}{*}{$\begin{array}{c}\text { Data Set } \\
\text { (2) }\end{array}$} & \multirow{2}{*}{$\begin{array}{c}\text { Julian Dates } \\
(-2,400,000) \\
(3)\end{array}$} & \multirow[b]{2}{*}{$\begin{array}{r}r_{\max } \\
(4)\end{array}$} & \multicolumn{2}{|c|}{$F_{\text {var }}$} & \multirow{2}{*}{$\begin{array}{c}\tau_{\text {cent }} \\
\text { (days) } \\
(7)\end{array}$} & \multirow{2}{*}{$\begin{array}{c}\tau_{\text {peak }} \\
\text { (days) } \\
(8)\end{array}$} \\
\hline & & & & $\begin{array}{c}\text { Continuum } \\
\text { (5) }\end{array}$ & $\begin{array}{c}\text { Line } \\
(6)\end{array}$ & & \\
\hline \multirow[t]{2}{*}{ 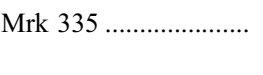 } & $\mathrm{H} \beta$ & $49,156-49,338$ & $0.829 \pm 0.066$ & 0.066 & 0.041 & $17.3_{-4.3}^{+4.9}$ & $18_{-6}^{+5}$ \\
\hline & $\mathrm{H} \beta$ & $49,889-50,118$ & $0.714 \pm 0.094$ & 0.049 & 0.015 & $12.8_{-5.7}^{+6.3}$ & $13_{-7}^{+9}$ \\
\hline \multirow[t]{2}{*}{ PG $0026+129 \ldots \ldots \ldots \ldots$} & $\mathrm{H} \alpha$ & $48,836-51,084$ & $0.796 \pm 0.047$ & 0.115 & 0.092 & $112.1_{-29.1}^{+32.3}$ & $108_{-36}^{+44}$ \\
\hline & $\mathrm{H} \beta$ & $48,545-51,084$ & $0.814 \pm 0.047$ & 0.173 & 0.079 & $126.8_{-32.3}^{+27.5}$ & $105_{-22}^{+37}$ \\
\hline \multirow[t]{3}{*}{ PG $0052+251 \ldots \ldots \ldots \ldots$} & $\mathrm{H} \alpha$ & $48,837-51,084$ & $0.646 \pm 0.063$ & 0.192 & 0.101 & $189.1_{-44.2}^{+67.6}$ & $194_{-89}^{+95}$ \\
\hline & $\mathrm{H} \beta$ & $48,461-51,084$ & $0.748 \pm 0.066$ & 0.199 & 0.114 & $103.7_{-27.8}^{+28.3}$ & $78_{-14}^{+19}$ \\
\hline & $\mathrm{H} \gamma$ & $48,461-51,084$ & $0.672 \pm 0.067$ & 0.199 & 0.199 & $94.2_{-18.4}^{+22.5}$ & $75_{-7}^{+12}$ \\
\hline \multirow[t]{3}{*}{ 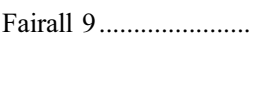 } & $\mathrm{H} \beta$ & $50,473-50,665$ & $0.715 \pm 0.067$ & 0.328 & 0.043 & $18.2_{-4.5}^{+3.3^{-4}}$ & $19_{-4}^{+3}$ \\
\hline & $\mathrm{C}_{\text {IV }} \lambda 1549$ & $50,473-50,713$ & $0.619 \pm 0.089$ & 0.385 & 0.056 & $: 31.0_{-15.1}^{+13.6}$ & $: 35_{-20}^{+14}$ \\
\hline & $\operatorname{Ly} \alpha$ & $50,473-50,713$ & $0.898 \pm 0.026$ & 0.385 & 0.115 & $12.5_{-5.9}^{+6.0}$ & $9_{-6}^{+6}$ \\
\hline
\end{tabular}

Noте.-Table 5 is also available in machine-readable form in the electronic edition of the Astrophysical Journal.

Peterson (2001). The estimates of the uncertainties are based on a model-independent Monte Carlo method in which a single realization yields a cross-correlation function (CCF) whose centroid $\tau_{\text {cent }}$, peak value $r_{\text {max }}$, and peak location $\tau_{\text {peak }}$ are measured. As discussed in the Appendix, we compute $\tau_{\text {cent }}$ using only the points at values $r \geq 0.8 r_{\max }$, where $r_{\max }$ is the peak value of the CCF. A large number of independent realizations is used to build up a "cross-correlation centroid distribution (CCCD)" and a "cross-correlation peak distribution (CCPD)" (see Maoz \& Netzer 1989). We take $\tau_{\text {cent }}$ and $\tau_{\text {peak }}$ to be the means of these distributions. The CCCDs and CCPDs are generally non-Gaussian, so we define upper and lower uncertainties separately such that $15.87 \%$ of the realizations yield values larger than the mean plus the upper error and that $15.87 \%$ of the realizations yield values smaller than the mean minus the lower error (i.e., the errors are $\pm 1 \sigma$ errors if the distribution is Gaussian).

We carried out a cross-correlation analysis for each data set, as summarized in Table 5. The object is listed in column (1) and the emission line and relevant Julian date range are listed in columns (2) and (3), respectively (see Table 2). Column (4) gives the peak amplitude of the CCF $r_{\max }$, and columns (5) and (6) give the noise-corrected fractional variation $F_{\text {var }}$ (see Rodríguez-Pascual et al. 1997) of the continuum and line, respectively, during the range of dates given in column (3). Columns (7) and (8) give the CCF centroid $\tau_{\text {cent }}$ and CCF peak $\tau_{\text {peak }}$, respectively, both in the observed frame. Uncertainties in these quantities were estimated by employing the modelindependent "flux randomization/random subset selection" (FR/RSS) method of P98b, with selected modifications suggested by Welsh (1999), as described in the Appendix. The uncertainty associated with $r_{\max }$ (col. [4]) is the rms variation in this quantity for the multiple Monte Carlo realizations. Note that entries preceded by colons are deemed to be unreliable (see the appropriate notes on the individual objects below), i.e., these are cases where there may be systematic uncertainties larger than indicated by the quoted uncertainties.

\section{TESTS OF VIRIAL RELATIONSHIPS}

Our next goal is to determine empirically which measures of time delay ( $\tau_{\text {cent }}$ or $\tau_{\text {peak }}$ ) and line width (FWHM or $\sigma_{\text {line }}$ ) provide the most robust estimates of the black hole masses. Specifically, we consider which combination of these measures gives us the most consistent or minimum variance virial product, $c \tau \Delta V^{2} / G$, where $\tau$ is the time delay and $\Delta V$ is the line width. This test can be performed on four of the objects in our sample for which long-duration multiwavelength spectroscopy allow measurements of a number of different variable emission lines. By far the best and most extensive data are those on NGC 5548, and we give these great weight in our analysis. On the other hand, we do not give much weight to the results for 3C 390.3 on account of relatively large uncertainties in both time lags and line widths.

Table 6 gives our measurements for the time-lag and linewidth parameters. Columns (1) and (2) identify the object and data set, in the same order as in Tables 2 and 5. Columns (3) and (4) contain $\tau_{\text {cent }}$ and $\tau_{\text {peak }}$, respectively; these are the values in Table 5 , now corrected for time dilation ${ }^{12}$ by dividing by $1+z$. Line width measurements were transformed to the rest frame of the object and converted to line-of-sight velocities; the values for $\sigma_{\text {line }}$ and FWHM are given in columns (5) and (6), respectively. Again, a colon preceding an entry indicates that we do not regard the entry to be reliable.

At this point, we make the assumption that the most robust measures of the time delay and line width are those that most closely yield the virial relationship $\Delta V \propto \tau^{-1 / 2}$. The justification for this assumption is simply that a virial relationship between time delay and line width has already been established for several objects (Peterson \& Wandel 1999, 2000; Onken \& Peterson 2002; Kollatschny 2003). We proceed by examining the four cases where multiple measurements of the virial product are available, giving the most weight to the results on NGC 5548.

\subsection{Virial Relationships in Individual Objects}

NGC 5548.-Figure 3 shows four plots of the virial relationship for all the lines in NGC 5548, as measured in the rms spectra. The optical data from the International AGN Watch (Peterson et al. 2002 and references therein) are divided into subsets based on single observing seasons separated by the several-week gap when NGC 5548 is too close to the Sun to observe. Some experimentation revealed that division into shorter subsets led to much larger errors in lag measurements. The four panels in Figure 3 show the possible permutations of

\footnotetext{
12 Paper I did not apply a time-dilation correction for their objects as the redshifts are all low. Kaspi et al. (2000) made this correction for their own higher redshift objects and the objects of Paper I.
} 
TABLE 6

Rest-Frame Lags, Line Widths, and Virial Products

\begin{tabular}{|c|c|c|c|c|c|c|}
\hline $\begin{array}{c}\text { Object } \\
\text { (1) }\end{array}$ & $\begin{array}{c}\text { Line } \\
(2)\end{array}$ & $\begin{array}{c}\tau_{\text {cent }} \\
\text { (days) } \\
\text { (3) }\end{array}$ & $\begin{array}{c}\tau_{\text {peak }} \\
\text { (days) } \\
\text { (4) }\end{array}$ & $\begin{array}{c}\sigma_{\text {line }} \\
\left(\mathrm{km} \mathrm{s}^{-1}\right) \\
(5)\end{array}$ & $\begin{array}{c}\text { FWHM } \\
\left(\mathrm{km} \mathrm{s}^{-1}\right) \\
(6)\end{array}$ & $\begin{array}{c}c \tau_{\text {cent }} \sigma^{2} \text { line } / G \\
\left(10^{6} M_{\odot}\right) \\
(7)\end{array}$ \\
\hline \multirow[t]{2}{*}{ Mrk 335} & $\mathrm{H} \beta$ & $16.8_{-4.2}^{+4.8}$ & $18_{-6}^{+5}$ & $917 \pm 52$ & $1629 \pm 145$ & $2.76_{-0.76}^{+0.85}$ \\
\hline & $\mathrm{H} \beta$ & $12.5_{-5.5}^{+6.6}$ & $13_{-7}^{+0}$ & $948 \pm 113$ & $1375 \pm 357$ & $2.20_{-1.10}^{+1.27}$ \\
\hline \multirow[t]{2}{*}{ PG $0026+129 \ldots \ldots \ldots \ldots \ldots \ldots \ldots$} & $\mathrm{H} \alpha$ & $98.1_{-25.5}^{+28.3}$ & $95_{-32}^{+39}$ & $1961 \pm 135$ & $1117 \pm 109$ & $73.7_{-21.7}^{+23.6}$ \\
\hline & $\mathrm{H} \beta$ & $111.0_{-28.3}^{+24.1}$ & $92_{-19}^{+32}$ & $1773 \pm 285$ & $1719 \pm 495$ & $68.2_{-28 .}^{+26.4}$ \\
\hline \multirow[t]{3}{*}{ 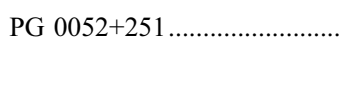 } & $\mathrm{H} \alpha$ & $163.7_{-38.3}^{+58.3}$ & $168_{-77}^{+82}$ & $1913 \pm 85$ & $: 2682 \pm 453$ & $117.0_{-29.3}^{+43.0}$ \\
\hline & $\mathrm{H} \beta$ & $89.8_{-24 .}^{+24.5}$ & $68_{-12}^{+16}$ & $1783 \pm 86$ & $4165 \pm 381$ & $55.7_{-15.9}^{+16.3}$ \\
\hline & $\mathrm{H} \gamma$ & $81.6_{-159}^{+19.5}$ & $65_{-6}^{-12}$ & $2230 \pm 502$ & $5633 \pm 3585$ & $79.2_{-38.8}^{+40.9}$ \\
\hline \multirow[t]{3}{*}{ 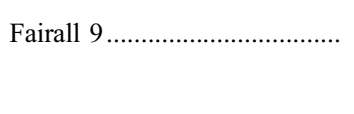 } & $\mathrm{H} \beta$ & $\begin{array}{l}01.0-15.9 \\
17.4_{-4.3}^{+3.2}\end{array}$ & $18_{-4}^{+3}$ & $3787 \pm 197$ & $6901 \pm 707$ & $48.7_{-130}^{+10.8}$ \\
\hline & $\mathrm{C}$ IV $\lambda 1549$ & $: 29.6_{-14.4}^{+12.3}$ & $: 33_{-19}^{+13}$ & $3201 \pm 285$ & $4628 \pm 1375$ & $\begin{array}{l}: 59.3_{-30.8}^{+28.0} \\
: 28\end{array}$ \\
\hline & Ly $\alpha$ & $11.9_{-5.6}^{+5.4}$ & $\begin{array}{c}-19 \\
9_{-6}^{+6}\end{array}$ & $4120 \pm 308$ & $3503 \pm 1474$ & $39.4_{-19.5}^{+20.8}$ \\
\hline
\end{tabular}

NoтE.-Table 6 is also available in machine-readable form in the electronic edition of the Astrophysical Journal.

the virial product using $\tau_{\text {cent }}$ and $\tau_{\text {peak }}$ for the time-delay parameter and $\sigma_{\text {line }}$ and FWHM as the line-width parameter. Fits to these data and the other virial relationships described below are summarized in Table 7, in each case for a best-fit slope and for a force-fit to a slope of $b=-\frac{1}{2}$. Fits were obtained using the orthogonal regression program GaussFit ${ }^{13}$ (Jefferys et al. 1988), which accounts for errors in both parameters. These data show clearly that (1) the virial relationship $\Delta V \propto \tau^{-1 / 2}$ is robust, i.e., precisely how the time delay and line width are measured is not critical, and (2) the least scatter in this relationship is obtained by using $\tau_{\text {cent }}$ and $\sigma_{\text {line }}$ to parameterize the relationship. This is confirmed by computing the virial product $c \tau \Delta V^{2} / G$ for each measure; the combination of $\tau_{\text {cent }}$ and $\sigma_{\text {line }}$ has a mean precision (standard deviation divided by the mean) of about 0.032 , which is lower than for the other pairs of measurements.

It is important to point out that the fractional errors in FWHM are rather larger than those in $\sigma_{\text {line. }}$ A simple consequence of this is that the $\chi^{2}$ statistic can be misleading, as it is larger for $\sigma_{\text {line }}$ than for FWHM. In any case, we hasten to point out that the scatter in these relationships is sufficiently large that it is clear that simple virial motion is an incomplete description of gas motions in the BLR. Figure 4 shows the virial relationship obtained by using the line widths measured from the mean spectra, uncorrected for narrow-line contamination. The deleterious effect of the narrow-line contribution on the line width measurements is most strongly apparent for $\mathrm{H} \beta$, as expected. In Figure 5 we show that correcting the spectra for narrow-line $\mathrm{H} \beta$ improves the result, but only somewhat. It seems clear that the rms spectrum should be used for these measurements.

NGC 3783 and NGC 7469.-Figures 6 and 7 show the virial relationship for NGC 3783 and NGC 7469 respectively, two of the other well-studied AGNs for which multiple measurements of the emission-line lags are available, although in both cases there are far fewer data than for NGC 5548. The measurements for NGC 3783 are in excellent agreement with viral relationship. The results for NGC 7469 are in poorer agreement, but the best-fit slope is within $2 \sigma$ of the virial prediction. Again our conclusions do not hinge critically on which time-lag and line-width measures we use.

3 C 390.3.-Figure 8 shows the case of 3C 390.3, which seems to afford some difficulties. The left-hand column shows

\footnotetext{
${ }^{13}$ GaussFit is publicly available at ftp://clyde.as.utexas.edu/pub/gaussfit.
}

a plot of line-width measures, $\sigma_{\text {line }}$ in the top panel and FWHM in the bottom panel, versus $\tau_{\text {cent }}$. The apparent lack of consistency with a virial relationship arises for the following reasons:

1. All the lines in this object are very broad, and each of the measured lines are contaminated by blending: Ly $\alpha$ is blended with $\mathrm{N} v \lambda 1240, \mathrm{C}$ IV $\lambda 1549$ is blended with $\mathrm{He}$ II $\lambda 1640$, and He II $\lambda 4686$ and $\mathrm{H} \beta$ are blended.

2. The emission-line lags have relatively large uncertainties and span a comparatively limited range (the largest and smallest lags differ by a factor of only $\sim 1.5$, compared to a factor of 7-14 for the other three objects discussed above).

In an attempt to circumvent the problem of line blending, we will assume that each line is intrinsically symmetric about its nominal wavelength. Its true width therefore can be better estimated by measuring only the unblended half of the line and reflecting it about the line center. The virial relationship using these line widths is replotted in the right-hand column of Figure 8. This gives somewhat improved consistency with the

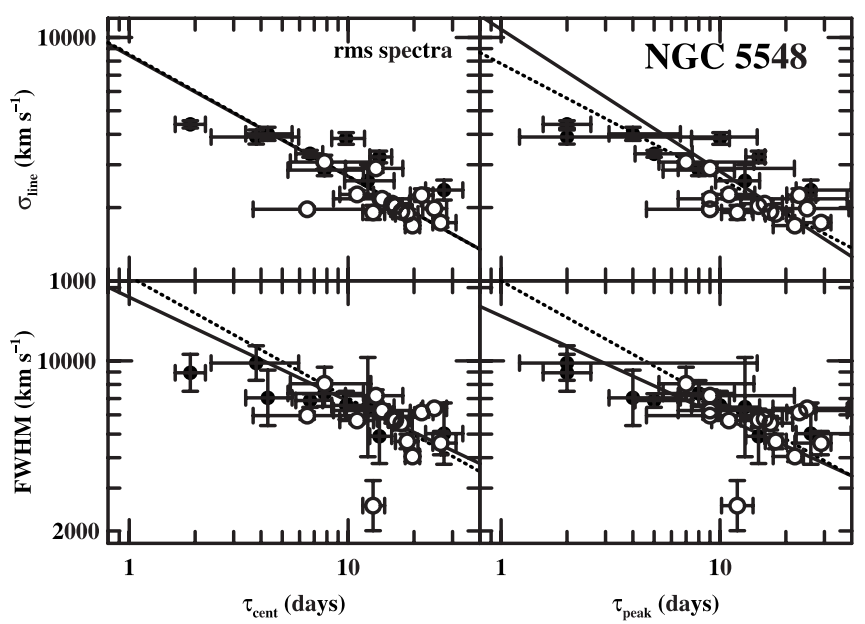

FIG. 3.-Line widths vs. time lags for emission lines in the rms spectra of NGC 5548. The top row shows the line dispersion $\sigma_{\text {line }}$ as a line width measure, and the bottom row shows FWHM. The left column shows the CCF centroid $\tau_{\text {cent }}$ as the time-lag measure and the right column shows the location of the CCF peak $\tau_{\text {peak }}$. The solid line is the best fit to the data, and the dotted line is a forced fit to slope $-\frac{1}{2}$, the virial slope. The fit parameters are summarized in Table 7. The open circles are measurements of $\mathrm{H} \beta$ for 14 different years. The filled circles represent all of the other lines. 
TABLE 7

Virial RELATIONShiP Fits

\begin{tabular}{|c|c|c|c|c|c|}
\hline \multirow[b]{2}{*}{ Data $S_{\text {et }}$} & \multicolumn{3}{|c|}{ Free SLOPE } & \multicolumn{2}{|c|}{ Fixed SLOPe $b=-0.5$} \\
\hline & $a$ & $b$ & $\chi_{\nu}^{2}$ & $a$ & $\chi_{\nu}^{2}$ \\
\hline \multicolumn{6}{|c|}{ NGC 5548: rms (Fig. 3) } \\
\hline$\sigma_{\text {line }}$ vs. $\tau_{\text {cent }} \ldots \ldots \ldots \ldots \ldots \ldots \ldots$ & $3.926 \pm 0.082$ & $-0.497 \pm 0.070$ & 4.04 & $3.930 \pm 0.016$ & 3.86 \\
\hline FWHM vs. $\tau_{\text {cent }} \ldots \ldots \ldots \ldots \ldots \ldots$ & $4.262 \pm 0.133$ & $-0.427 \pm 0.112$ & 4.25 & $4.346 \pm 0.021$ & 4.12 \\
\hline 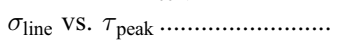 & $4.033 \pm 0.138$ & $-0.583 \pm 0.117$ & 2.96 & $3.936 \pm 0.018$ & 2.88 \\
\hline 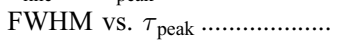 & $4.187 \pm 0.102$ & $-0.378 \pm 0.086$ & 2.09 & $4.328 \pm 0.020$ & 2.13 \\
\hline \multicolumn{6}{|c|}{ NGC 5548: Mean ${ }^{a}$ (Fig. 4) } \\
\hline$\sigma_{\text {line }}$ vs. $\tau_{\text {cent }} \ldots \ldots \ldots \ldots \ldots \ldots \ldots$ & $4.058 \pm 0.135$ & $-0.545 \pm 0.114$ & 7.60 & $4.005 \pm 0.017$ & 7.30 \\
\hline FWHM vs. $\tau_{\text {cent }} \ldots \ldots \ldots \ldots \ldots \ldots$ & $6.202 \pm 1.602$ & $-2.277 \pm 1.357$ & 14.42 & $4.108 \pm 0.041$ & 29.15 \\
\hline 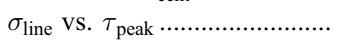 & $4.264 \pm 0.268$ & $-0.721 \pm 0.228$ & 4.25 & $4.005 \pm 0.020$ & 4.44 \\
\hline 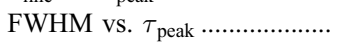 & $8.035 \pm 3.761$ & $-3.882 \pm 3.209$ & 5.95 & $4.081 \pm 0.046$ & 19.23 \\
\hline \multicolumn{6}{|c|}{ NGC 5548: Mean' (Fig. 5) } \\
\hline$\sigma_{\text {line }}$ vs. $\tau_{\text {cent }} \ldots \ldots \ldots$ & $3.917 \pm 0.072$ & $-0.422 \pm 0.060$ & 4.70 & $4.009 \pm 0.014$ & 4.74 \\
\hline 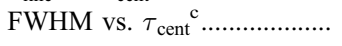 & $3.870 \pm 0.066$ & $-0.088 \pm 0.060$ & 70.25 & $4.341 \pm 0.033$ & 54.68 \\
\hline 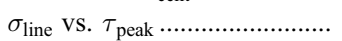 & $3.987 \pm 0.112$ & $-0.483 \pm 0.095$ & 2.78 & $4.006 \pm 0.015$ & 2.65 \\
\hline 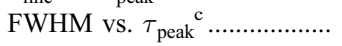 & $3.871 \pm 0.057$ & $-0.092 \pm 0.053$ & 46.90 & $4.328 \pm 0.034$ & 31.11 \\
\hline \multicolumn{6}{|c|}{ NGC 3783: rms (Fig. 6) } \\
\hline$\sigma_{\text {line }}$ vs. $\tau_{\text {cent }} \ldots \ldots$. & $3.690 \pm 0.042$ & $-0.428 \pm 0.063$ & 0.28 & $3.735 \pm 0.021$ & 0.28 \\
\hline 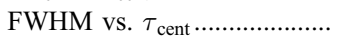 & $3.925 \pm 0.098$ & $-0.497 \pm 0.144$ & 0.74 & $3.927 \pm 0.037$ & 0.50 \\
\hline 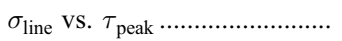 & $3.696 \pm 0.078$ & $-0.446 \pm 0.122$ & 0.52 & $3.727 \pm 0.030$ & 0.37 \\
\hline 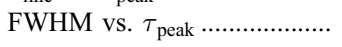 & $3.894 \pm 0.069$ & $-0.473 \pm 0.104$ & 0.25 & $3.910 \pm 0.026$ & 0.17 \\
\hline \multicolumn{6}{|c|}{ NGC 7469: rms (Fig. 7) } \\
\hline 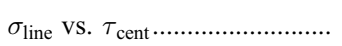 & $3.786 \pm 0.114$ & $-0.952 \pm 0.248$ & 1.19 & $3.592 \pm 0.042$ & 3.36 \\
\hline 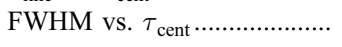 & $4.066 \pm 0.093$ & $-1.119 \pm 0.195$ & 0.50 & $3.772 \pm 0.067$ & 3.94 \\
\hline 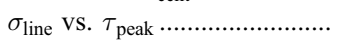 & $3.737 \pm 0.105$ & $-0.918 \pm 0.209$ & 0.69 & $3.553 \pm 0.049$ & 2.32 \\
\hline 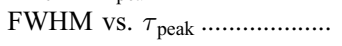 & $4.028 \pm 0.084$ & $-1.113 \pm 0.164$ & 0.28 & $3.742 \pm 0.073$ & 3.36 \\
\hline \multicolumn{6}{|c|}{ 3C 390.3: rms ${ }^{\text {d }}$ (Fig. 8) } \\
\hline 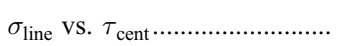 & $2.083 \pm 1.027$ & $+1.009 \pm 0.686$ & 0.72 & $4.339 \pm 0.097$ & 5.09 \\
\hline FWHM vs. $\tau_{\text {cent }} \ldots \ldots \ldots \ldots \ldots \ldots \ldots$ & $4.818 \pm 0.991$ & $-0.566 \pm 0.659$ & 1.20 & $4.718 \pm 0.045$ & 0.80 \\
\hline \multicolumn{6}{|c|}{ 3C 390.3: $\mathrm{rms}^{\mathrm{e}}$ (Fig. 8) } \\
\hline$\sigma_{\text {line }}$ vs. $\tau_{\text {cent }} \ldots \ldots \ldots \ldots \ldots \ldots \ldots \ldots$ & $2.660 \pm 0.547$ & $+0.598 \pm 0.365$ & 0.64 & $4.300 \pm 0.076$ & 3.10 \\
\hline 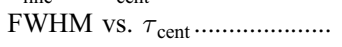 & $4.197 \pm 0.108$ & $-0.149 \pm 0.070$ & 0.13 & $4.723 \pm 0.036$ & 0.39 \\
\hline
\end{tabular}

${ }^{\text {a }}$ Includes narrow-line components.

${ }^{b}$ Narrow component of $\mathrm{H} \beta$ removed.

c Free-slope fit is unweighted.

${ }^{\mathrm{d}}$ Full line width.

e Unblended wing of line.

virial relationship, especially for FWHM. However, the relatively low range in lags and line widths and large errors in lag measurements hardly make this object a convincing case for a virial relationship. Given the large systematic uncertainties in the line widths on account of line blending and the relatively poor precision of time-delay measurements we do not believe that these results are inconsistent with a virial relationship.

\subsection{General Results}

We conclude from this analysis that the most consistent virial product is obtained by using $\tau_{\text {cent }}$ and $\sigma_{\text {line }}$ as the timelag and line-width measures. The discussion in the Appendix further assures us that $\tau_{\text {cent }}$ is a good choice for the lag mea- surement. The virial product computed from $\tau_{\text {cent }}$ and $\sigma_{\text {line }}$ is thus given for each data set in column (7) of Table 6.

We also conclude that for the purpose of determining black hole masses, the rms spectrum provides the most reliable line width measurement. However, it is also clear (e.g., Fig. 5) that the mean spectrum (or perhaps even a single spectrum), with its much higher signal-to-noise ratio than the rms spectrum, can be used with little penalty in accuracy, as long as one can adequately account for contamination by other features, notably the narrow-line contribution and blending with adjacent features. However, the strength of narrow-line contributions to broadline spectra are often known rather poorly, if at all, and blending by various features, notably $\mathrm{Fe}$ II contamination of $\mathrm{H} \beta$, is 


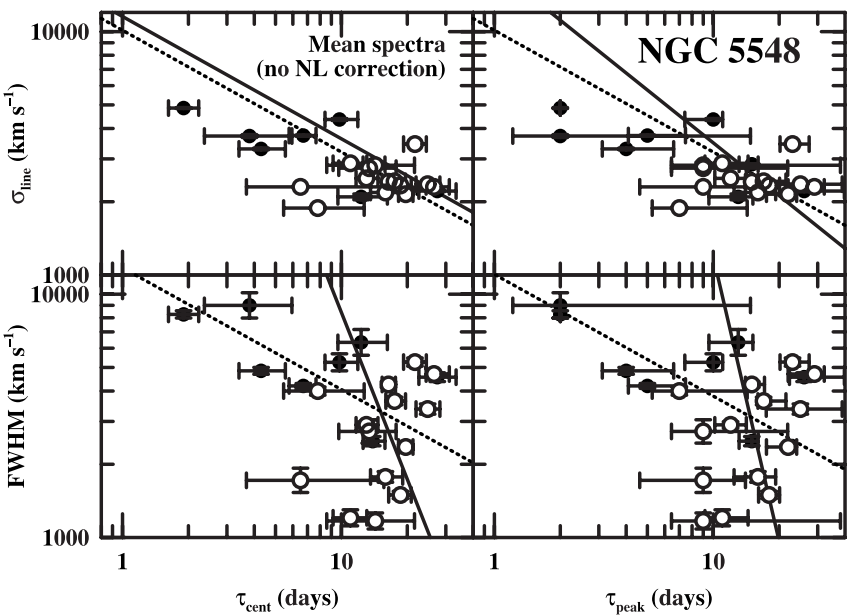

FIG. 4.- Line width vs. time lags for emission lines in the mean spectra of NGC 5548. The data are plotted as in Fig. 3. Note the dramatic change in the scatter, especially for the $\mathrm{H} \beta$ line, by using the mean rather than the rms line profile for the line-width measurement.

problematic. Further discussion is beyond the scope of the current paper but will be pursued elsewhere.

For completeness, we show in Figures 9 and 10 the distribution of the ratios FWHM $/ \sigma_{\text {line }}$ and $\tau_{\text {peak }} / \tau_{\text {cent }}$, respectively, for all lines used in this analysis (i.e., highly uncertain values excluded). The mean and standard deviations of these distributions are $\mathrm{FWHM} / \sigma_{\text {line }}=2.03 \pm 0.59$ and $\tau_{\text {peak }} / \tau_{\text {cent }}=0.95 \pm$ 0.20 . The low mean value of FWHM $/ \sigma_{\text {line }}$ relative to that for a Gaussian $\left(\mathrm{FWHM} / \sigma_{\text {line }}=2.355\right)$ means that, on average, these lines have weaker cores and stronger wings relative to Gaussians.

\section{BLACK HOLE MASSES}

For many of the objects in this study, we have multiple measurements of the virial products. In Table 8, we list for each object in this study the weighted mean virial product $\left\langle c \tau_{\text {cent }} \sigma_{\text {line }}^{2} / G\right\rangle$, based on the entries in column (7) of Table 6 ,

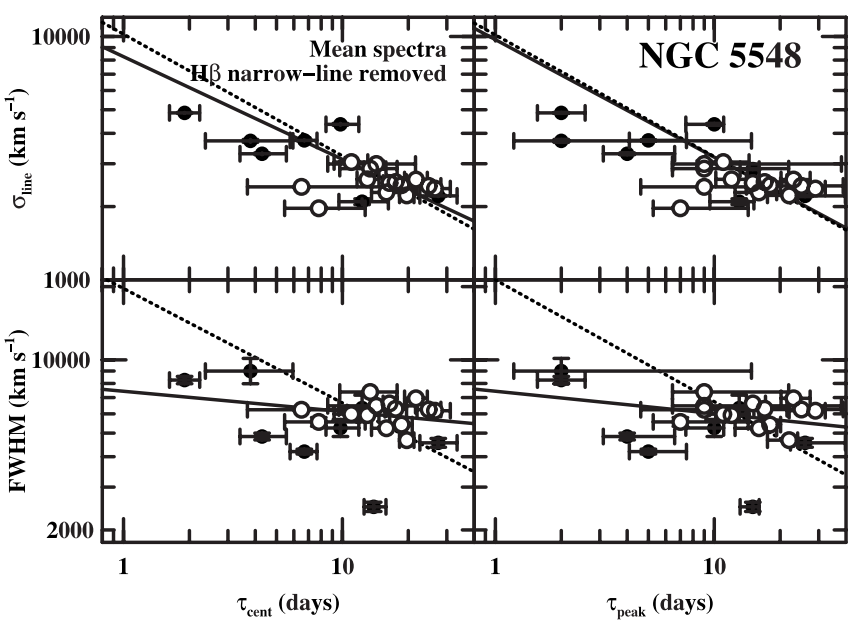

FIG. 5.-Line widths vs. time lags for emission lines in the mean spectra of NGC 5548, but with the narrow component of $\mathrm{H} \beta$ removed. The data are plotted as in Figs. 3 and 4. Removing the narrow component of $\mathrm{H} \beta$ greatly reduces the scatter around the virial relationship, but the scatter is still much more pronounced than in Fig. 3, where the line widths are measured in the rms profiles. The measurement uncertainties on $\sigma_{\text {line }}$ are too small to show up on this diagram. Note that the fits to the data in the lower panel are unweighted fits on account of the large number of outliers.

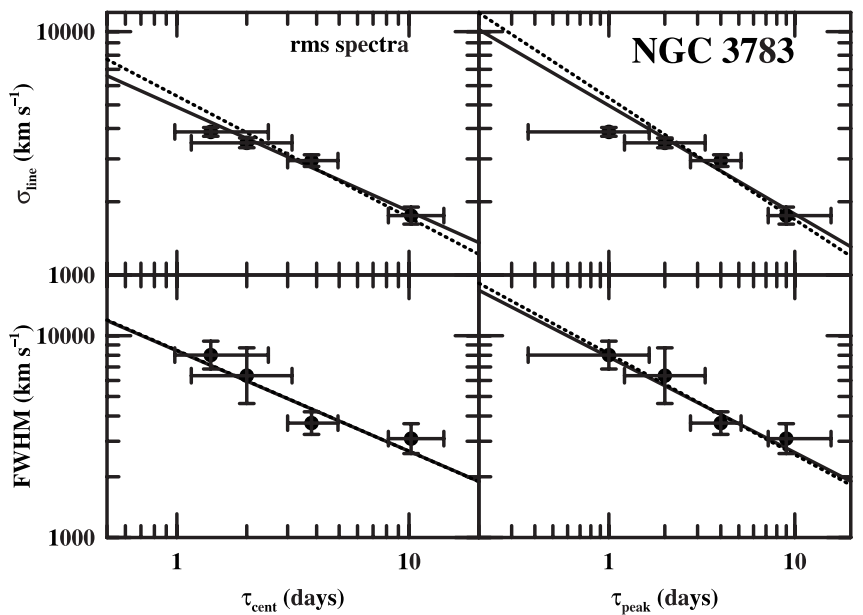

FIG. 6.-Line widths vs. time lags for emission lines in the rms spectra of NGC 3783. The data are plotted as in Fig. 3.

excluding the more uncertain values (those preceded by a colon).

The masses of the central objects are given by

$$
M_{\mathrm{BH}}=\frac{f_{c} \tau_{\text {cent }} \sigma_{\text {line }}^{2}}{G},
$$

where as noted earlier $f$ depends on the structure, kinematics, and aspect of the BLR. The scaling factor $f$ can be determined in a number of ways, the easiest being to assume that AGNs and quiescent galaxies follow the same $\mathrm{M}_{\mathrm{BH}}-\sigma_{*}$ relationship; one can then use quiescent galaxy results to normalize the AGN $\mathrm{M}_{\mathrm{BH}^{-}} \sigma_{*}$ relationship and provide an absolute mass scale for AGN black holes. We carry out this exercise in a companion paper (Onken et al. 2004), in which we find $\langle f\rangle=5.5$. Our final black hole masses, based on equation (7) with an adopted mean value $\langle f\rangle=5.5$, are given in column (3) of Table 8 .

\subsection{Uncertainties in Black Hole Masses}

As noted earlier, the first goal of this project has been to improve the precision of the virial product measurement. We find that the typical precision (i.e., fractional error) of the virial product measurement is about $33 \%$ for the 35 AGNs for which we are able to estimate black hole masses, or $\sim 26 \%$, excluding

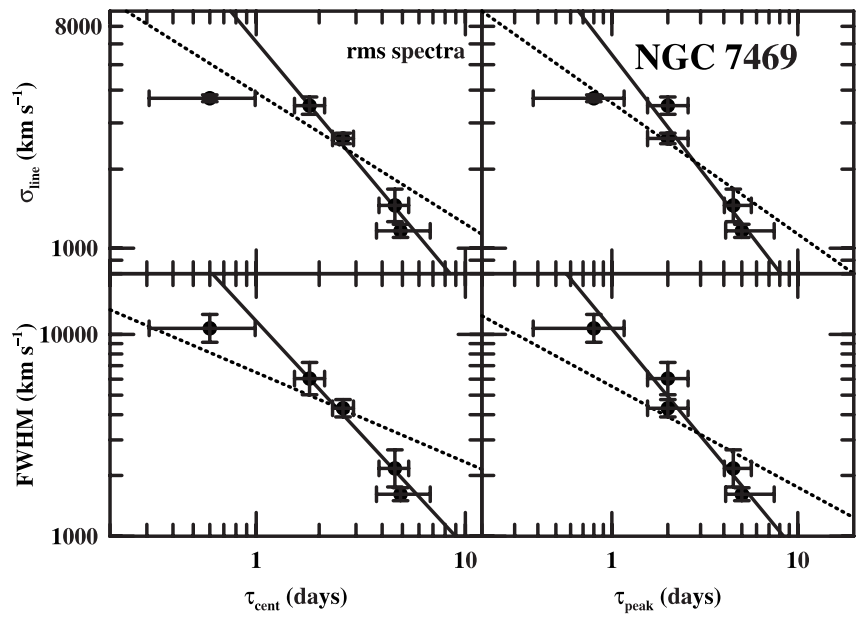

FIG. 7.-Line widths vs. time lags for emission lines in the rms spectra of NGC 7469. The data are plotted as in Fig. 3. 


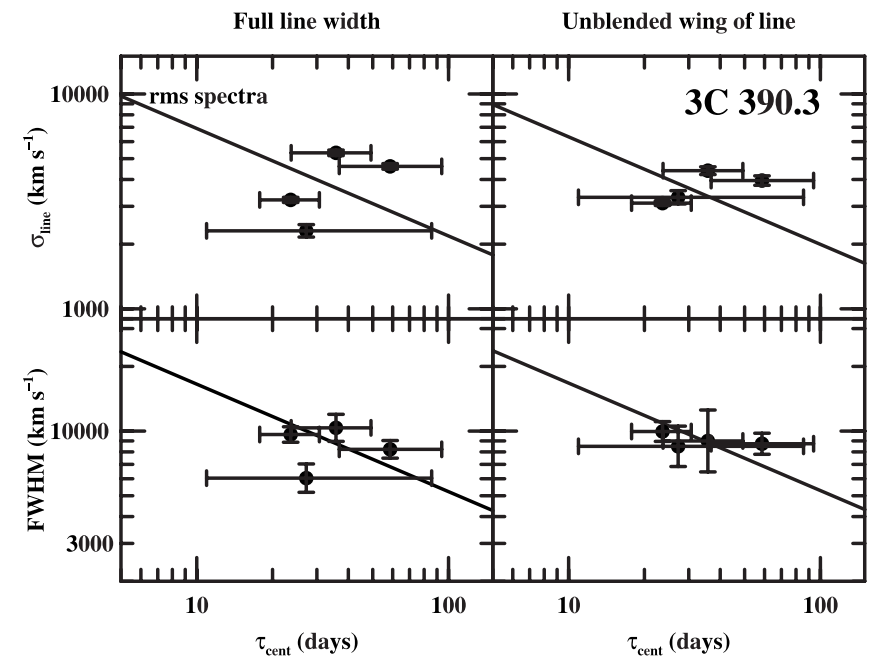

FIG. 8.- Line width vs. CCF centroid $\tau_{\text {cent }}$ for emission lines in the rms spectra of 3 C 390.3. The top row shows the line dispersion $\sigma_{\text {line }}$ used as the linewidth measure, and the bottom row shows FWHM. The left-hand column shows our standard line-width measurement, using the whole line, but with no effort to account for blending. In the right-hand column, we attempt to account for blending by measuring the unblended half of each line. In any case, the large uncertainties in the time lags preclude a critical test of a virial relationship.

NGC 4593 and IC 4329A, for which the reverberation results are notably poor. The second goal is to improve the statistical accuracy of the reverberation-based black hole mass scale using the normalization of the AGN $M_{\mathrm{BH}} \sigma_{*}$ relationship reported in a companion paper (Onken et al. 2004). The scatter around the AGN $M_{\mathrm{BH}^{-}} \sigma_{*}$ relationship is found to be about a factor of 2.62.9 , depending somewhat on the slope of the quiescent galaxy $M_{\mathrm{BH}^{-}} \sigma_{*}$ relationship (Onken et al. 2004). It is important to keep in mind that this level of accuracy is statistical in nature and individual black hole masses may be less accurate.

It must be kept in mind that there are various systematic difficulties with reverberation results that can in principle lead

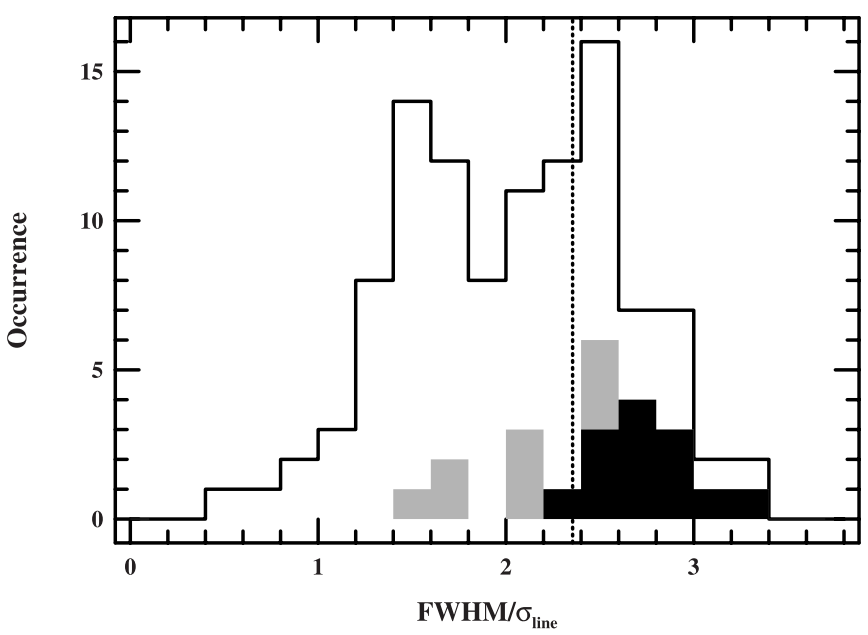

FIG. 9.-Distribution of values of FWHM/ $/ \sigma_{\text {line }}$ for all lines in the rms spectra used in this analysis (i.e., cols. [5] and [(6] of Table 6), excluding highly uncertain values (preceded with a colon in Table 6). The mean and standard deviation for the total distribution are $2.03 \pm 0.59$. The black area shows the distribution for multiple measurements of $\mathrm{H} \beta$ in NGC 5548 (the mean and standard deviation for this subset are $2.73 \pm 0.24)$. The gray area shows the distribution for other lines in NGC 5548 (mean and standard deviation for all lines in NGC 5548, including $\mathrm{H} \beta$, are $2.45 \pm 0.44$. The vertical dotted line is at $\mathrm{FWHM} / \sigma_{\text {line }}=2.355$, which is appropriate for a Gaussian line profile. Values smaller than this indicate lines that have weaker cores and strong wings relative to a Gaussian.

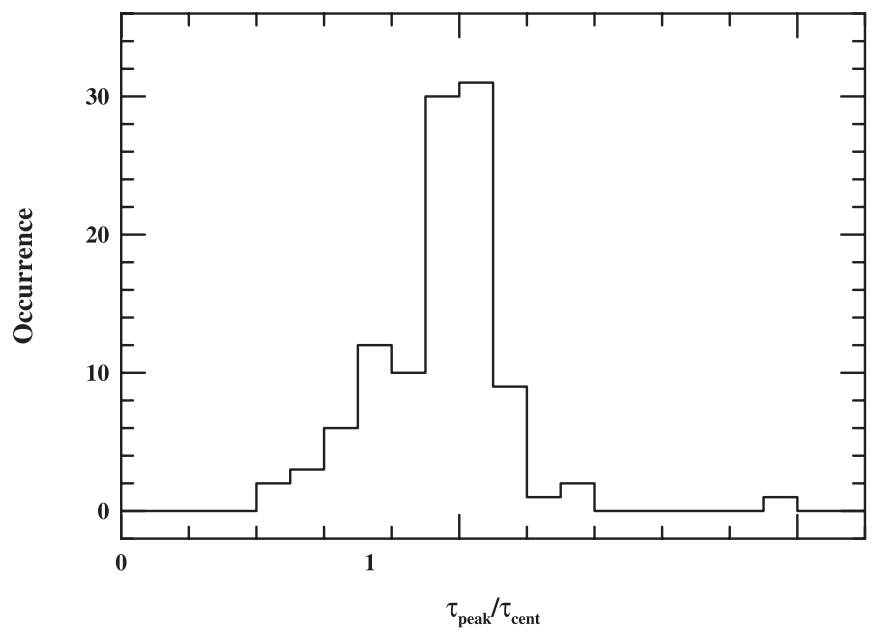

FIG. 10.-Distribution of values of $\tau_{\text {peak }} / \tau_{\text {cent }}$ for all lines in this analysis (i.e., cols. [(3] and [(4] of Table 6), excluding highly uncertain values (preceded with a colon in Table 6). The mean and standard deviation for this distribution are $0.95 \pm 0.20$.

TABLE 8

Adopted Virial Products and Derived Black Hole Masses

\begin{tabular}{|c|c|c|c|}
\hline $\begin{array}{c}\text { Object } \\
\text { (1) }\end{array}$ & $\begin{array}{c}\left\langle c \tau_{\text {cent }} \sigma_{\text {line }}^{2} / G\right\rangle \\
\left(10^{6} M_{\odot}\right) \\
(2)\end{array}$ & $\begin{array}{c}M_{\mathrm{BH}}^{\mathrm{a}} \\
\left(10^{6} M_{\odot}\right) \\
(3)\end{array}$ & $\begin{array}{c}\log \lambda L_{\lambda} \text { (optical) } \\
\left(\operatorname{ergs~s}^{-1}\right) \\
(4)\end{array}$ \\
\hline Mrk 335 . & $2.58 \pm 0.67$ & $14.2 \pm 3.7$ & $43.86 \pm 0.04$ \\
\hline PG $0026+129$. & $71.4 \pm 17.4$ & $393 \pm 96$ & $45.02 \pm 0.06$ \\
\hline PG $0052+251$. & $67.1 \pm 13.8$ & $369 \pm 76$ & $44.96 \pm 0.08$ \\
\hline Fairall $9 \ldots$ & $46.3 \pm 10.1$ & $255 \pm 56$ & $44.25 \pm 0.05$ \\
\hline Mrk 590 . & $8.64 \pm 1.34$ & $47.5 \pm 7.4$ & $43.81 \pm 0.09$ \\
\hline 3C $120 \ldots \ldots \ldots \ldots \ldots \ldots \ldots$ & $10.1_{-4.1}^{+5.7}$ & $55.5_{-22.5}^{+31.4}$ & $44.17 \pm 0.08$ \\
\hline Ark 120. & $27.2 \pm 3.5$ & $150 \pm 19$ & $44.23 \pm 0.09$ \\
\hline Mrk 79 & $9.52 \pm 2.61$ & $52.4 \pm 14.4$ & $43.72 \pm 0.05$ \\
\hline PG $0804+761$. & $126 \pm 15$ & $693 \pm 83$ & $44.94 \pm 0.08$ \\
\hline PG $0844+349$. & $16.80 \pm 7.06$ & $92.4 \pm 38.1$ & $44.35 \pm 0.04$ \\
\hline Mrk $110 \ldots \ldots \ldots \ldots$ & $4.57 \pm 1.1$ & $25.1 \pm 6.1$ & $43.72 \pm 0.09$ \\
\hline PG $0953+414 \ldots$. & $50.1 \pm 10.7$ & $276 \pm 59$ & $45.22 \pm 0.06$ \\
\hline NGC $3227 \ldots$. & $7.67 \pm 3.90$ & $42.2 \pm 21.4$ & $42.38 \pm 0.04$ \\
\hline NGC $3516 .$. & $7.76 \pm 2.65$ & $42.7 \pm 14.6$ & $42.88 \pm 0.13$ \\
\hline NGC $3783 \ldots .$. & $5.42 \pm 0.99$ & $29.8 \pm 5.4$ & $43.26 \pm 0.04$ \\
\hline NGC $4051 .$. & $0.348 \pm 0.142$ & $1.91 \pm 0.78$ & $41.93 \pm 0.03$ \\
\hline NGC $4151 \ldots .$. & $2.42 \pm 0.83$ & $13.3 \pm 4.6$ & $42.88 \pm 0.23$ \\
\hline PG $1211+143$. & $: 26.6 \pm 8.0$ & $: 146 \pm 44$ & $44.75 \pm 0.07$ \\
\hline PG $1226+023 .$. & $161 \pm 34$ & $886 \pm 187$ & $45.96 \pm 0.05$ \\
\hline PG $1229+204 \ldots \ldots \ldots \ldots . . . .$. & $13.3 \pm 6.4$ & $73.2 \pm 35.2$ & $44.08 \pm 0.05$ \\
\hline NGC $4593 \ldots \ldots \ldots$. & $0.975_{-1.264}^{+1.704}$ & $5.36_{-6.95}^{+9.37}$ & $43.09 \pm 0.14$ \\
\hline PG $1307+085 \ldots$ & $80.0 \pm 22.3$ & $440 \pm 123$ & $44.88 \pm 0.04$ \\
\hline IC 4329A ….................. & $: 1.80_{-2.16}^{+3.25}$ & $: 9.90_{-11.88}^{+17.88}$ & $43.32 \pm 0.05$ \\
\hline Mrk 279 .......................... & $6.35 \pm 1.67$ & $34.9 \pm 9.2$ & $43.88 \pm 0.05$ \\
\hline PG $1411+442$. & $80.5 \pm 26.5$ & $443 \pm 146$ & $44.63 \pm 0.04$ \\
\hline NGC $5548 \ldots . .$. & $12.20 \pm 0.47$ & $67.1 \pm 2.6$ & $43.51 \pm 0.11$ \\
\hline PG $1426+015$. & $236 \pm 70$ & $1298 \pm 385$ & $44.72 \pm 0.07$ \\
\hline Mrk $817 \ldots \ldots \ldots . .$. & $8.98 \pm 1.40$ & $49.4 \pm 7.7$ & $43.82 \pm 0.05$ \\
\hline PG $1613+658 \ldots \ldots \ldots \ldots . . . . .$. & $50.7_{-23.5}^{+23.4}$ & $279 \pm 129$ & $44.98 \pm 0.05$ \\
\hline PG $1617+175 \ldots \ldots \ldots \ldots \ldots$ & $108 \pm 25$ & $594 \pm 138$ & $44.48 \pm 0.08$ \\
\hline PG $1700+518 \ldots \ldots \ldots \ldots \ldots$ & $142_{-30}^{+33}$ & $781_{-165}^{+182}$ & $45.63 \pm 0.03$ \\
\hline 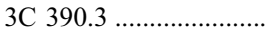 & $52.2 \pm 11.7$ & $287 \pm 64$ & $43.95 \pm 0.07$ \\
\hline Mrk 509 ......................... & $26.0 \pm 2.1$ & $143 \pm 12$ & $44.28 \pm 0.08$ \\
\hline PG $2130+099 \ldots \ldots \ldots \ldots . . . . .$. & $83.0 \pm 10.0$ & $457 \pm 55$ & $44.46 \pm 0.04$ \\
\hline NGC $7469 \ldots \ldots \ldots \ldots \ldots \ldots$ & $2.21 \pm 0.25$ & $12.2 \pm 1.4$ & $43.72 \pm 0.02$ \\
\hline
\end{tabular}

a Assuming $f=5.5$. 
to significant errors in individual black hole masses (e.g., Krolik 2001). Reverberation, of course, is not the only method of measuring black hole masses that can fail catastrophically under certain conditions: both stellar dynamical and gas dynamical methods can also lead to ambiguous or even misleading results (e.g., Verdoes Kleijn et al. 2000; Cappelari et al. 2002; Valluri et al. 2004). While we find the limited scatter in the AGN $M_{\mathrm{BH}^{-}} \sigma_{*}$ relationship reassuring, additional tests remain highly desirable.

\subsection{On Normalization of the $A G N M_{\mathrm{BH}^{-}} \sigma_{*}$ Relationship}

As noted above, we normalize the AGN $M_{\mathrm{BH}^{-}} \sigma_{*}$ relationship to the $M_{\mathrm{BH}}-\sigma_{*}$ relationship for quiescent galaxies by setting $f=$ 5.5 in equation (7). This represents the first empirical determination of the zero point in the AGN black hole mass scale.

In previous work the scale factor appeared in a different and more model-dependent way, only as an adjustment $\epsilon$ to the line-width parameter, i.e.,

$$
\sigma=\epsilon \mathrm{FWHM}
$$

and the virial mass is $M=c\langle\tau\rangle \sigma^{2} / G$. For example, Netzer (1990) assumed $\epsilon=\sqrt{3} / 2$, which arises from assuming that $\sigma_{\text {line }}=\mathrm{FWHM} / 2$, and that the velocity dispersion is isotropic, i.e., $\sigma=\sqrt{3} \sigma_{\text {line }}$, recalling that $\sigma_{\text {line }}$ is the line-of-sight velocity dispersion. This further assumes that the mean time delay $\langle\tau\rangle$ is independent of aspect or inclination, which is true when the line emission is isotropic and unabsorbed and the geometry has spherical or polar symmetry. If we make the equivalent assumptions in equation (7), i.e., that the velocity dispersion is isotropic so $\sigma=\sqrt{3} \sigma_{\text {line }}$ and that $\sigma_{\text {line }} \approx \mathrm{FWHM} / 2$ (which is on average quite a good assumption, given the results of the previous section), then $f=3$. Our empirical calibration of the AGN mass scale through the AGN $M_{\mathrm{BH}^{-}} \sigma_{*}$ relationship is thus about a factor of $1.8(=5.5 / 3)$ higher than the mass scale used in previous papers (e.g., Paper I and Kaspi et al. 2000). We emphasize again that the previous normalization was made only in the absence of observational information or better justified assumptions; the value we give here is the first determination based on observational parameters, in this case as embodied in the $M_{\mathrm{BH}}-\sigma_{*}$ relationship.

\section{COMMENTS ON SELECTED INDIVIDUAL OBJECTS}

$P G 0026+129$. - Because of strong narrow-line residuals in the rms spectrum, we found it necessary to remove the narrow component of $\mathrm{H} \beta$ and the $[\mathrm{O}$ III] $\lambda \lambda 4959,5007$ lines from the individual spectra. However, the narrow lines in the vicinity of $\mathrm{H} \alpha$ are sufficiently weak that they present no problems.

$P G \quad 0052+251$. - The narrow-line components were removed from the $\mathrm{H} \beta$ region, but the $\mathrm{H} \alpha$ region suffers from significant narrow-line residuals in the rms spectrum. The FWHM of $\mathrm{H} \alpha$ is untrustworthy and probably underestimated for this reason.

Fairall 9.- In this case, we used the time-binned UV continuum light curve at $1390 \AA$ (Rodríguez-Pascual et al. 1997) as the driving light curve for all lines, including $\mathrm{H} \beta$. Unfortunately, the $\mathrm{C}$ IV $\lambda 1549$ light curve is very noisy, and the crosscorrelation result is not trustworthy. It is therefore excluded from the mass determination.

Mrk 590.- The $\mathrm{H} \beta$ profile in the rms spectrum in the first data set (JD 2,448,090-2,448,323) seems anomalously narrow, although the rms spectrum for this data set is significantly noisier than for the other rms spectra (note the lower value of $F_{\text {var }}$ in Table 5). We have therefore excluded the first data set

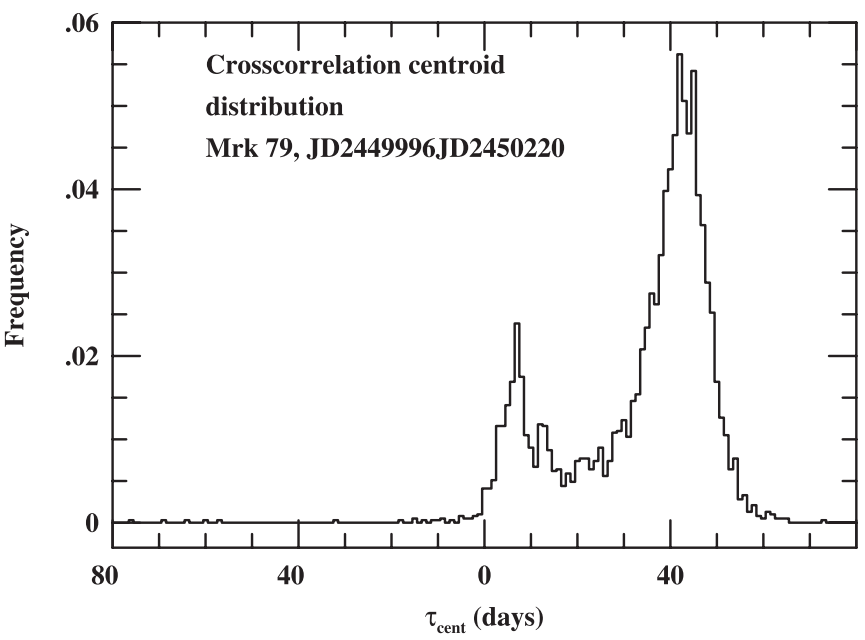

FIG. 11.-Cross-correlation centroid distribution for the continuum-H $\beta$ cross-correlation for Mrk 79 during the period JD 2,449,996-2,450,220. It is not obvious which peak corresponds to the correct lag.

from the mass analysis, since the line width is questionable although the lag measurement seems trustworthy.

Mrk 79.- The lag measurements from the fourth data set (JD 2,449,996-2,450,220) are not trustworthy on account of some significant aliasing effects, seen clearly in the doublepeaked CCCD shown in Figure 11. We have therefore excluded this data set.

$P G 0804+761$. - This is one of a handful of objects that are similar to the well-known Seyfert galaxy I Zw 1. These "I Zw 1-like" AGNs have relatively narrow lines and very strong optical Fe II emission; they are among the more extreme members of the narrow-line Seyfert 1 (NLS1) subclass. A particular problem these objects present is that the [O III] $\lambda \lambda 4959$, 5007 lines are heavily contaminated by $\mathrm{Fe}$ II emission, and in fact, most of the emission that makes up these features can be attributed to $\mathrm{Fe}$ II $\lambda 4924$ and Fe II $\lambda 5018$ (e.g., Peterson et al. 1984). We are thus unable to use [O III] $\lambda 5007$ as a template for removal of the narrow-line contaminants. However, in these cases, narrow $\mathrm{H} \beta$ is usually too weak to strongly affect the linewidth measurements, so we do not attempt narrow-line removal for these objects. In this particular quasar, there is a clear residual narrow-line $\mathrm{H} \alpha$ component in the rms spectrum, but the line-width measurement is probably not strongly affected. On the other hand, a strong [O III] $\lambda 4363$ residual makes $\mathrm{H} \gamma$ highly asymmetric in the rms spectrum, and thus the line-width measurements for $\mathrm{H} \gamma$ cannot be trusted.

$P G$ 0844+349. - This is another I Zw 1-like object (see PG 0804+761 above). The time-lag measurements are very inconsistent from line-to-line, probably because of inadequate time sampling. There are narrow-line residuals in $\mathrm{H} \gamma$ and $\mathrm{H} \alpha$. The $\mathrm{H} \beta$ cross-correlation function is clearly strongly affected by correlated errors and is thus rejected. The results on this object are of rather low quality.

Mrk 110.-The $\mathrm{H} \beta$ data used here are from Peterson et al. (1998a). He II $\lambda 4686$ appears as a prominent, broad feature in the rms spectra of this object, as shown in Figure 12. We therefore constructed a light curve for $\mathrm{He}$ II from the original data. Unfortunately, the He II lags are so short that the measurements of $\tau_{\text {cent }}$ and $\tau_{\text {peak }}$ cannot be trusted, as they are significantly shorter than the time interval between observations. We therefore do not use the He II lines in the mass determination, although we point out that they are generally consistent with the $\mathrm{H} \beta$ results. Kollatschny (2003) has also studied the 


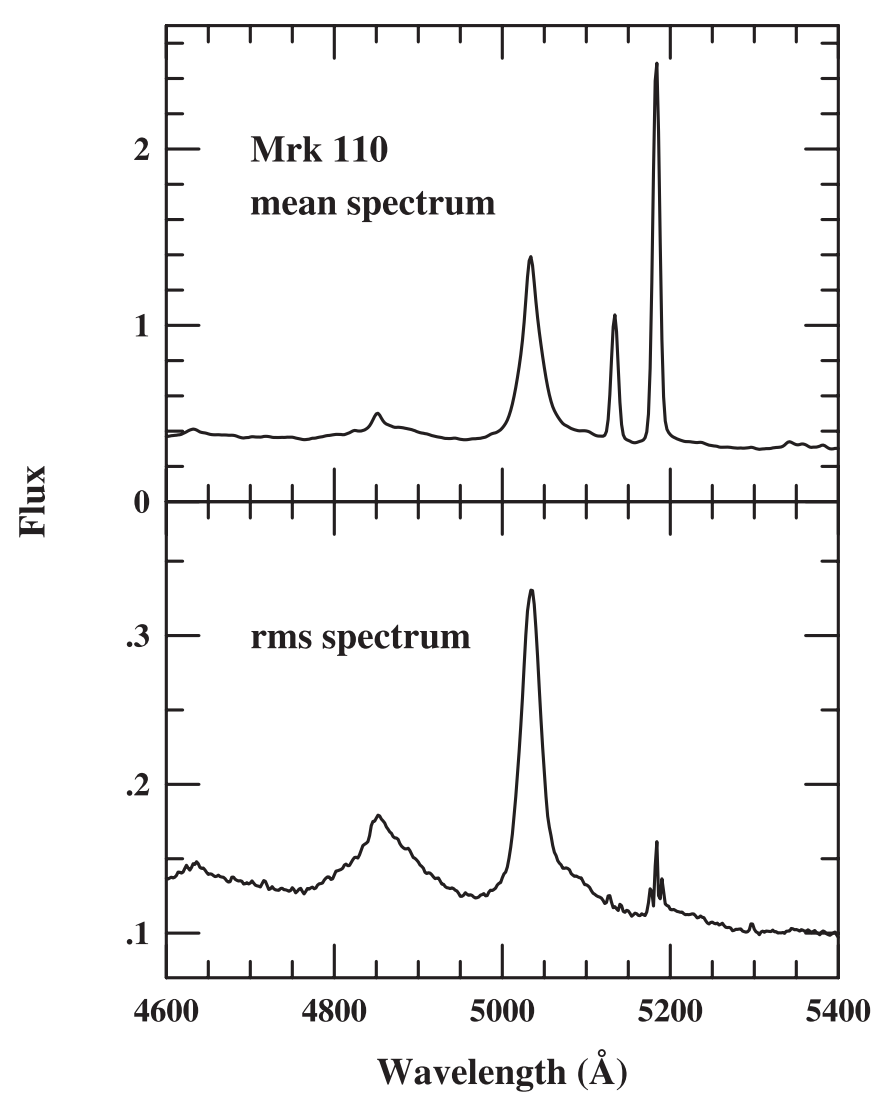

FIG. 12.-Mean (top) and rms (bottom) spectra of Mrk 110. The rms spectrum shows clearly the very broad $\mathrm{He}$ II $\lambda 4686$ and broad $\mathrm{H} \beta$. The narrow [O III] $\lambda \lambda 4959,5007$ lines appear only in the mean spectrum, except for weak residuals in [O III] $\lambda 5007$ that appear in the rms spectrum.

variability of this $\mathrm{AGN}$ and finds that the results for $\mathrm{H} \alpha, \mathrm{H} \beta$, He I $\lambda 5876$, and He II $\lambda 4686$ are consistent with a single virial mass. As discussed in more detail by Onken et al. (2004), the black hole mass measured by Kollatschny is consistent with our measurement if we use FWHM instead of $\sigma_{\text {line }}$ and Kollatschny's value for the scaling factor $f$.

$N G C$ 3227.- This AGN was the target of two separate optical campaigns, one by the LAG consortium in 1990 (Salamanca et al. 1994) and one at CTIO in 1992 (Winge et al. 1995). The rms spectra formed from the LAG data were recently presented by Onken et al. (2003). We completely reanalyzed the CTIO data. The original reduced spectra were rescaled in flux using the van Groningen \& Wanders (1992) algorithm that has been used in most of the International AGN Watch campaigns and in the Ohio State program, and new continuum and $\mathrm{H} \beta$ emission-line light curves were measured from the rescaled spectra. While this resulted in some improvement in the $\mathrm{H} \beta$ lag determination and uncertainty, the rms spectrum was still quite noisy because of the combination of a low amplitude of variability and a relatively insensitive detector (see the notes on IC 4392A, below).

$N G C$ 3516. - This is another object observed by the LAG consortium (Wanders et al. 1993). We note that the light curves for this object may be less reliable than those of other objects as the extended narrow-line region in this object makes narrow line-based flux calibration vulnerable to seeing effects, which then have to be modeled (Wanders et al. 1992). We used the scaled and corrected spectra to determine the rms spectrum, following Onken et al. (2003).

$N G C$ 3783. - This was the second major multiwavelength campaign undertaken by the International AGN Watch

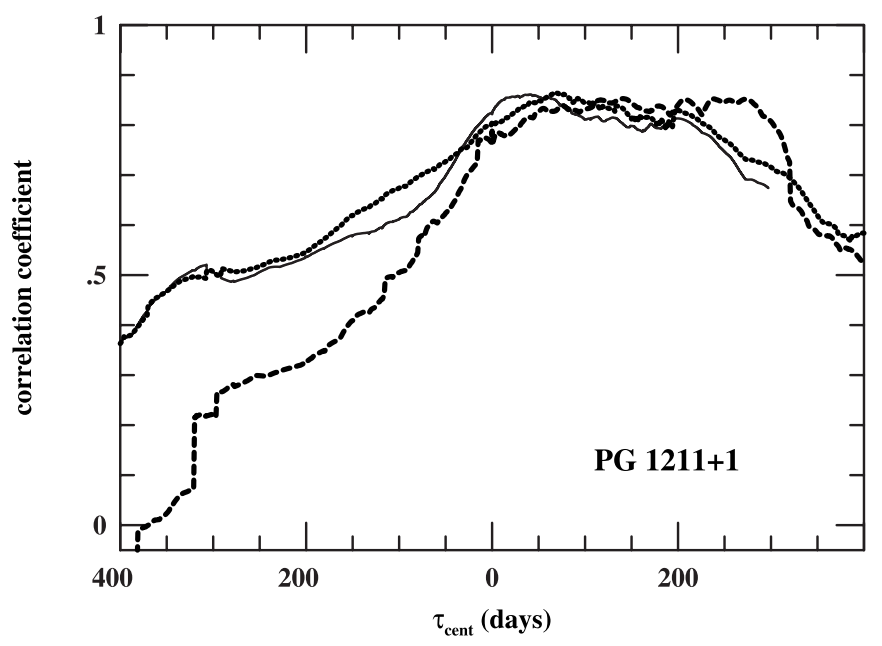

FIG. 13.-Cross-correlation functions for PG 1211+143. The dotted line is the $\mathrm{H} \alpha \mathrm{CCF}$, the solid line is the $\mathrm{H} \beta \mathrm{CCF}$, and the dashed line is the $\mathrm{H} \gamma \mathrm{CCF}$. The centroids and peaks are poorly defined because the CCFs are broad and flat-topped.

(Reichert et al. 1994; Stirpe et al. 1994). This object was completely reanalyzed by Onken \& Peterson (2002); new UV light curves based on IUE NEWSIPS data were measured, and the optical spectra were completely recalibrated using the van Groningen \& Wanders (1992) algorithm. The results presented here are based on the Onken \& Peterson reanalysis.

NGC 4051.- This object was also studied recently by Shemmer et al. (2003), who obtain a black hole mass consistent with the results of Peterson et al. (2000), who present the data we have used here.

$N G C$ 4151. - We analyze two sets of optical data on this object, from the Wise Observatory campaign in 1988 (Maoz et al. 1991) and from the International AGN Watch project in 1993-1994 (Kaspi et al. 1996). Unfortunately, the rms line profiles in the 1988 data are too poor to use due to a combination of narrow-line residuals in the rms spectra, uncertain narrow-line removal from the original spectra, and a variable line-spread function. We therefore do not use these data, except to the extent of noting that the results are broadly consistent with the later AGN Watch results; although the lag measurements appear to be reliable, the line widths in the rms spectra are not trustworthy.

$P G 1211+143$. - This AGN is another I Zw 1-like object (see PG 0804+761 above). Our level of confidence in the results for this object is low: the lags are suspect because the amplitude of variability is low, the variations are slow, and the time sampling is not especially good. The time lags are highly uncertain on account of this; the CCFs are very flat-topped and uncertain, as can be seen clearly in Figure 13. Moreover, there are some problems with stability in the $\mathrm{H} \alpha$ region of the spectrum that makes the line-width measurements of $\mathrm{H} \alpha$ highly uncertain. Given these difficulties, we exclude this object from further analysis.

$P G 1226+023$.- This is the well-known quasar, 3C 273, which is another I Zw 1-like object (see PG 0804+761 above) in which the [O III] lines are strongly blended with Fe II lines (Peterson et al. 1984). The narrow-line components of $\mathrm{H} \gamma$ and $\mathrm{H} \beta$ are weak in both the mean and rms spectra. However, there are strong narrow-line residuals in the $\mathrm{H} \alpha$ region, so the $\mathrm{H} \alpha$ line-width measurements cannot be trusted.

$P G 1229+204$. - Inspection of the light curves shows that the variations in this object are not well sampled. The large 


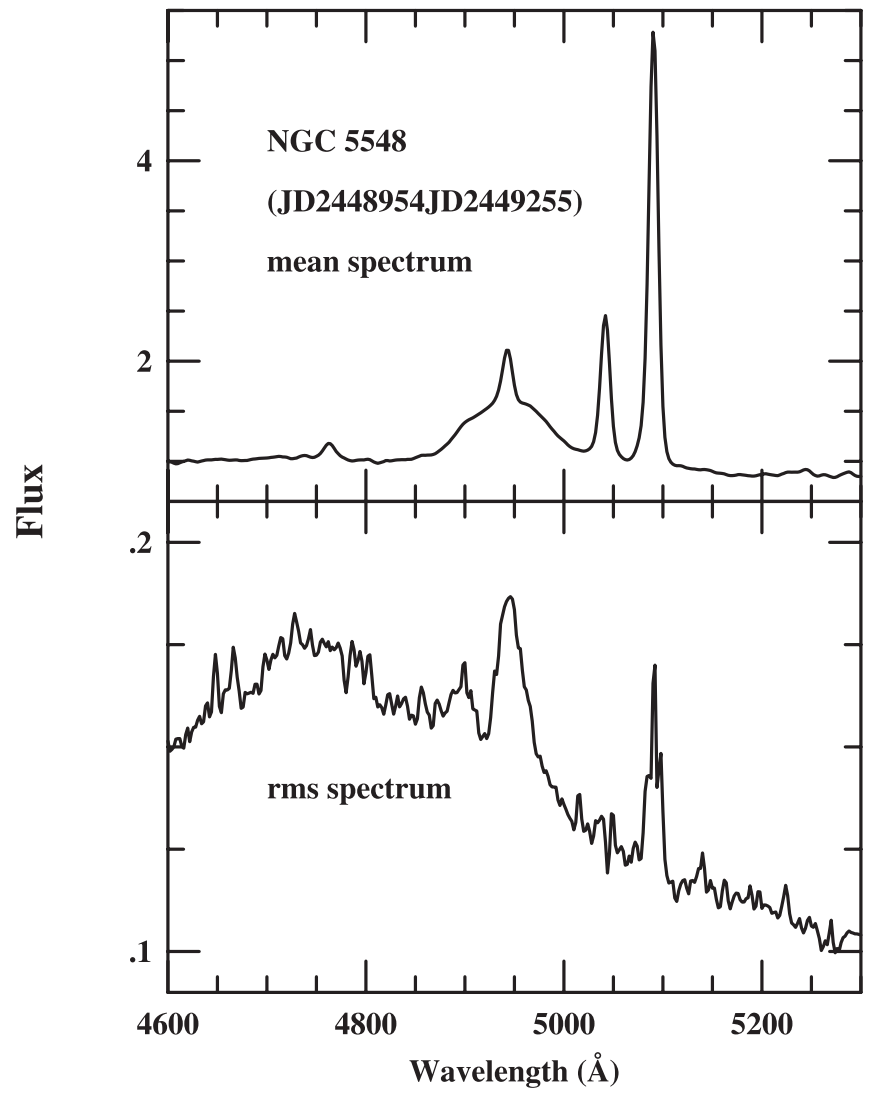

FIG. 14.-Mean (top) and rms (bottom) $\mathrm{H} \beta$-region spectra of NGC 5548 during the period JD 2,448,954-2,449,255, the fifth year of monitoring by the International AGN Watch (1993). The $\mathrm{H} \beta$ profile in the rms spectrum is double-peaked; there is a strong peak at line center and another in the shortward wing of the line.

differences in lags for the Balmer lines make the results on this object rather dubious. We have low confidence in the results for this object.

$N G C$ 4593.- This is another object from the LAG campaign in 1990 (Dietrich et al. 1994), where the data have been reanalyzed by Onken et al. (2003). We regard the $\mathrm{H} \beta$ lag as completely unreliable because it is so much smaller than the mean sampling interval. The $\mathrm{H} \alpha$ lag should also be regarded with some caution.

$P G 1307+085$. - The $\mathrm{H} \alpha$ region of the rms spectrum in this object shows residual narrow-line $\mathrm{H} \alpha$, although this probably affects only the FWHM measurement.

IC 4329A.- This object and NGC 3227 were both observed in the CTIO monitoring program (Winge et al. 1995, 1996). These observations employed a Reticon detector, which yielded poorer quality spectra than obtained with the CCDs used in virtually every other ground-based campaign. We attempted to improve the original light curves by rescaling the original spectra in flux by using the van Groningen \& Wanders (1992) algorithm and remeasuring the continuum and emission-line fluxes. This did improve light curves and rms spectra, but only marginally. The light curves are very poor, and the time-lag measurements should be regarded with caution. The FWHM measurement is very poor (note the large uncertainty yielded by our measurement algorithm). We have little confidence in the mass determination for this object.

Mrk 279.-We examined two completely independent sets of data, one from the Wise Observatory program in 1988 ( Maoz et al. 1990) and one from an International AGN Watch project

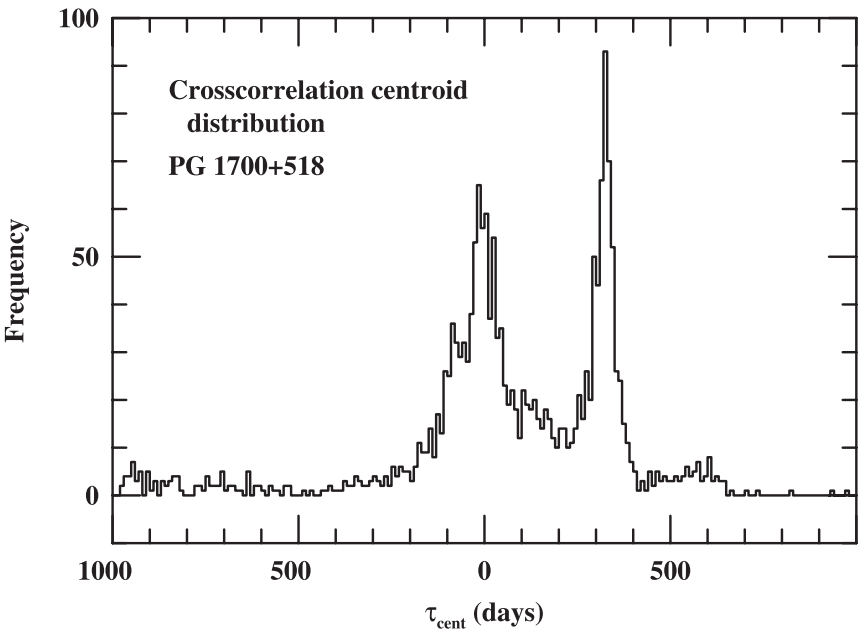

FIG. 15.-Cross-correlation centroid distribution for the continuum-H $\beta$ cross-correlation for PG $1700+518$. The peak at zero lag is clearly ascribable to correlated error, so it can be rejected.

in 1996 (Santos-Lleó et al. 2001). The AGN Watch data appear to be quite good. The Wise Observatory data, however, have a number of problems. The CCFs for both $\mathrm{H} \alpha$ and $\mathrm{H} \beta$ show a correlated error signal at zero lag; ${ }^{14}$ this problem is particularly bad at $\mathrm{H} \beta$, and we will therefore not use the $\mathrm{H} \beta$ lag measurements. Furthermore, the $\mathrm{H} \alpha$ region of the rms spectrum is strongly contaminated by narrow-line residuals, and $\mathrm{H} \beta$ is so weak that the FWHM measurement is meaningless. Nevertheless, the mass determination is quite consistent with the results of the AGN Watch program.

NGC 5548.- Some comments on this object appear in $\S 5$. There are more published variability data, by far, on this object than any other, and most of the data are very good. Only a few problems need to be pointed out. First, the $\mathrm{H} \alpha$ region of the rms spectrum from the Wise Observatory campaign (Netzer et al. 1990) has strong narrow-line residuals that render the line-width measures unusable. The CCCD for the associated time series is also rather ambiguous, so we do not include these measurements in the mass determination. The $\mathrm{H} \beta$ profile in the rms spectrum from the fifth year of AGN Watch monitoring (i.e., JD $2,448,954-2,449,255)$ is very unusual, as shown in Figure 14; there are two peaks, a strong central peak and a weak blue peak. The FWHM measurement is thus meaningless. Finally, He II $\lambda 4686$ is prominent in the rms spectrum for the first year of the AGN Watch campaign (JD 2,447,509$2,447,809)$, but is too heavily blended with the optical Fe II blends to measure in the mean spectrum; this line is not included in this part of the analysis (see Figs. 4 and 5).

$P G$ 1700+518. - The CCCD for this object shows two peaks, one of them clearly ascribable to correlated errors in the continuum and emission-line light curves (Fig. 15). Fortunately, the peaks are well-separated, and we can exclude the zero-lag correlated error peak from our analysis. This significantly increases the $\mathrm{H} \beta$ lag and also the black hole mass relative to the original investigation by Kaspi et al. (2000).

3 C 390.3.- Some of the difficulties with this object have already been discussed in $\S 5$. Part of the problem with these data seems to be just the nature of the variability: apart from a

\footnotetext{
14 Correlated errors result from flux calibration problems. An error in flux calibration offsets both the line and continuum measurements based on that spectrum in the same direction, thus introducing a spurious cross-correlation signal at zero lag.
} 
large-scale outburst during the early part of the monitoring campaign, the variations were very weak. As noted earlier, the lines are very broad and blended, and the most consistent black hole mass is obtained by using the line width measured from the unblended side of each line and then assuming symmetry. A more detailed attempt at deconvolution might improve this. Finally, we note that in this case, we used the UV continuum (at $1370 \AA$ ) as the driving continuum in the cross-correlation analysis.

Mrk 509.-Like Mrk 110, the He II $\lambda 4686$ line is prominent in the rms spectrum of this object, so we therefore attempted to measure it in each spectrum and produce a light curve. Because of blending with optical Fe II emission, the resulting light curve is probably not as reliable as the $\mathrm{H} \beta$ light curve.

$N G C 7469$. - This object is one of a very few in which a lag has been detected between the UV and optical continuum variations (Wanders et al. 1997; Collier et al. 1998). We therefore in this case use the UV continuum (at $1315 \AA$ ) as the driving continuum in the cross-correlation analysis.

\section{THE MASS-LUMINOSITY RELATIONSHIP}

Our improved database can be used to investigate the relationships between BLR radius and luminosity and black hole mass and luminosity. The radius-luminosity relationship is discussed in a companion paper (S. Kaspi et al. 2004, in preparation), and here we will discuss only the mass-luminosity relationship.

We computed the optical luminosity from the flux measurements in the original data sources that were made over the time intervals used in this analysis. In each case, we selected the continuum waveband closest to $5100 \AA$ in the AGN rest frame. We corrected for Galactic reddening using the extinction values in column (7) of Table 1 and using the reddening curve of Savage \& Mathis (1979), adjusted to $A_{V} / E(B-V)=3.2$. Luminosity distances were computed using the redshifts given in column (5) of Table 1 and by assuming a standard flat $\Lambda \mathrm{CDM}$ cosmology with $\Omega_{B}=0.04, \Omega_{\mathrm{DM}}=0.26, \Omega_{\Lambda}=0.70$, and $H_{0}=70 \mathrm{~km} \mathrm{~s}^{-1} \mathrm{Mpc}^{-1}$. For AGNs with multiple mass determinations, we computed the average value of $\log \lambda L_{\lambda}$ from each individual time series. The optical luminosities $\log \lambda L_{\lambda}$ are given in column (4) of Table 8 . The corresponding uncertainties represent the amplitude of continuum variability during the reverberation experiment.

In Figure 16 we plot the reverberation-based masses we have derived as a function of the mean luminosity. This figure can be compared directly with Figure 8 of Kaspi et al. (2000), which reveals that a much better defined mass-luminosity relationship results from our improved analysis. We have also estimated the bolometric luminosity in the same fashion as Kaspi et al., i.e., $L_{\text {bol }} \approx 9 \lambda L_{\lambda}(5100 \AA)$, and this scale is shown on the top of Figure 16. The diagonal lines show the Eddington limit and $10 \%$ and $1 \%$ its value.

We note that this constitutes only a preliminary version of the mass-luminosity relationship, for comparison with earlier work. A more exhaustive study of this relationship is continuing. Several comments are in order:

1. It is reassuring that there are no objects above the Eddington limit (i.e., to the right of the diagonal line), in contrast to the results of Kaspi et al. This is because our analysis has corrected a number of errors in earlier work, most notably removal of residual narrow-line $\mathrm{H} \beta$ from many of the rms spectra, which resulted in larger line widths and rather higher overall black hole masses. The object closest to the Eddington limit is, not surprisingly, 3C 273 (PG 1226+023).

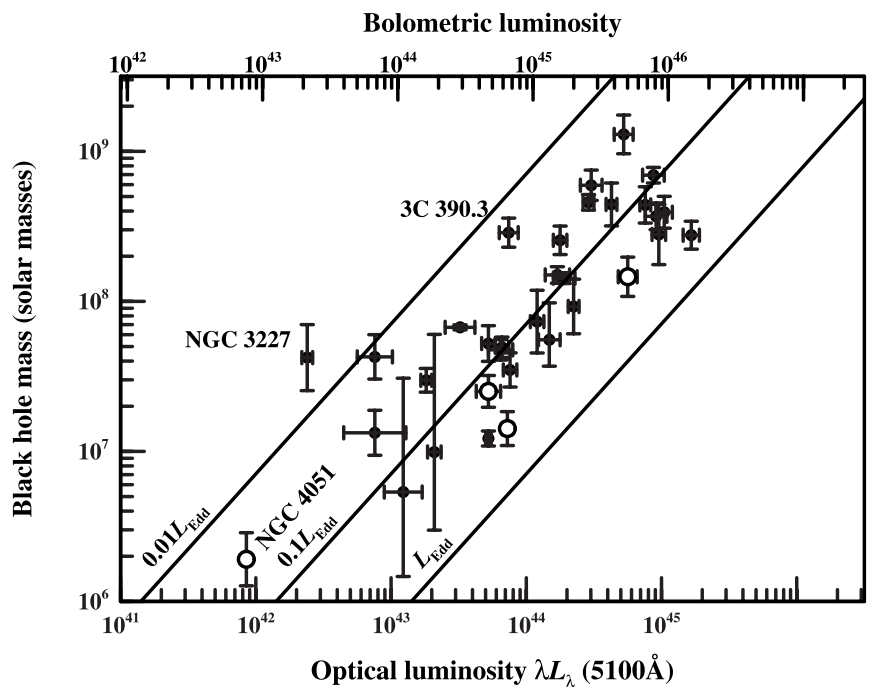

FIG. 16.-Black hole mass vs. luminosity for 35 reverberation mapped AGNs. The luminosity scale on the lower $x$-axis is $\log \lambda L_{\lambda}$ in units of $\operatorname{ergs~s}{ }^{-1}$. The upper $x$-axis shows the bolometric luminosity assuming that $L_{\mathrm{bol}} \approx 9 \lambda L_{\lambda}$. The diagonal lines show the Eddington limit $L_{\text {Edd }}, 0.1 L_{\text {Edd }}$, and $0.01 L_{\text {Edd }}$. The open circles represent NLS1s. Other labeled points are discussed in the text.

2. The bolometric correction we have assumed is nominal, based on a spectral energy distribution (SED) with a strong blue bump. However, observed SEDs suggest that a smaller ratio, e.g., $L_{\text {bol }} \approx 5 \lambda L_{\lambda}$ (Netzer 2003 ), may be on average more appropriate. Moreover, the bolometric correction we adopt may not be appropriate for all types of AGNs at arbitrary luminosity. The bolometric luminosities are thus uncertain and should be treated with some caution.

3. The optical luminosities used here have not been corrected for the contribution of starlight from the host galaxies. This can be a significant factor, especially in the lower luminosity objects. For example, the standard aperture used in the NGC 5548 optical monitoring program, 5. $0 \times 7$ ".5, admits a starlight flux of $F_{\lambda}(5100 \AA) \approx 3.4 \times 10^{-15} \operatorname{ergs~s}{ }^{-1} \mathrm{~cm}^{-2} \AA^{-1}$ (Romanishin et al. 1995). Correcting for this reduces the optical luminosity entry for NGC 5548 in Table 8 by $\sim 0.24$ dex. A program is currently underway to determine the starlight contribution to the optical luminosity of each of these objects.

4. Internal extinction has not been accounted for in any way. Correction for extinction will move objects to the right in this diagram. It is worth noting that the object with the lowest Eddington rate (farthest to the left of the $0.01 L_{\text {Edd }}$ diagonal) is NGC 3227, which is a rather dusty object (Pogge \& Martini 2002). The low luminosity of this object relative to its black hole mass may be a result of internal extinction.

5. Given the small formal error bars for most of the objects, we believe that much of the scatter in Figure 16 is real. Moreover, we find that the scatter correlates with other AGN properties; the I $\mathrm{Zw} \mathrm{1-type} \mathrm{objects} \mathrm{generally} \mathrm{lie} \mathrm{along} \mathrm{the} \mathrm{bottom}$ edge of the envelope defined by the data points. In Figure 16 the NLS1s are shown as open circles, and all of them except NGC 4051 lie on the lower edge of the mass-luminosity envelope. Conversely, the one object with strongly double-peaked Balmer line profiles, 3C 390.3, lies along the upper edge of the envelope. The locations of these extreme objects on this diagram suggest that at least some of the dispersion of the data points correlates with eigenvector 1 , consistent with the suggestion originated by Boroson \& Green (1992) and reaffirmed by numerous later authors that eigenvector 1 appears to be driven by 
Eddington ratio $\dot{m}=\dot{M} / \dot{M}_{\text {Edd }}$. However, the physical origin of the scatter observed in Figure 16 could be attributable either to differences in Eddington ratio or to inclination effects. Decreasing inclination (i.e., from edge-on to face-on) will translate points to the right as the apparent luminosity increases on account of decreased limb darkening and downward as the rotational velocities appear to decrease. Increasing the Eddington ratio will translate points in the same sense.

6. The best fit mass-luminosity relationship is found to be

$$
\begin{aligned}
\log \left(M / 10^{8} M_{\odot}\right) & =-0.12( \pm 0.07) \\
+ & 0.79( \pm 0.09) \log \left(\lambda L_{\lambda}(5100 \AA) / 10^{44} \mathrm{erg} \mathrm{s}^{-1}\right) .
\end{aligned}
$$

However, there is no reason to believe that there are no selection effects operating. Interestingly, the lower edge of the envelope seems to parallel the lines of constant Eddingtion ratio rather well, suggesting that the intrinsic mass-luminosity slope may not differ significantly from unity.

\section{SUMMARY}

In this contribution we have improved the calibration of the reverberation-based AGN black hole mass scale by decreasing random and systematic errors and by drawing on the AGN $M_{\mathrm{BH}^{-}} \sigma_{*}$ relationship to establish a statistically accurate calibration that is tied to other methods of black hole mass measurement. We have undertaken a consistent reanalysis of a highly inhomogeneous database that consists of 117 separate time series, not including several others that were deemed to be too poor to use, in the process accounting for a variety of systematic effects such as time dilation on the time lags and spectral resolution on the line widths. Each time series is treated independently and yields an independent estimate of the black hole mass, thus reducing random uncertainties. Poor or suspicious data are removed from the database, noting especially the susceptibility of time-lag measurements to correlated errors and other types of aliasing. We find that the most consistent mass measurements are obtained by using the cross-correlation centroid $\tau_{\text {cent }}$ to characterize the light-travel time across the BLR and by using the line dispersion $\sigma_{\text {line }}$ as measured in the rms spectrum to characterize the velocity dispersion of the BLR. In practice, special care has been taken to remove residual narrowline contamination of the rms spectrum in cases where it is present. The result of this analysis is a revised AGN mass scale based on 35 reverberation-mapped AGNs that is statistically accurate to better than a factor of 3 .

We are grateful for support of this work through NSF grant AST 02-05964 to The Ohio State University, through USIsrael Binational Science Foundation grant 1999-336, and through a grant from Israel Science Foundation grant 232/03. This research has made use of the NASA/IPAC Extragalactic Database (NED), which is operated by the Jet Propulsion Laboratory, California Institute of Technology, under contract with the National Aeronautics and Space Administration. We thank an anonymous referee for suggestions that led to improvements in the paper.

\section{APPENDIX}

\section{SOME COMMENTS ON CROSS-CORRELATION METHODOLOGY}

Welsh (1999) has suggested a number of modifications to our error analysis procedures, and we have investigated these through additional Monte Carlo simulations similar to those described by P98b.

Emission-line lag measurements are made by cross-correlating the emission-line and continuum light curves. Since the data are almost never regularly sampled, real data points in one time series are matched with values obtained by linear interpolation of the other time series. The cross-correlation function (CCF) is measured twice, once interpolating in the continuum series, and once in the emission-line series, and the final CCF is determined by averaging these two results. The CCF is characterized by (1) its peak value (highest value of the correlation coefficient), $r_{\max },(2)$ the time delay corresponding to this value, $\tau_{\text {peak }}$, and (3) the centroid $\tau_{\text {cent }}$ of the peak in the CCF. In practice, $\tau_{\text {cent }}$ is evaluated using only points with values about some threshold, usually $0.8 r_{\max }$.

To assess the uncertainties in the determination of $\tau_{\text {peak }}$ and $\tau_{\text {cent }}$, we use the model-independent Monte Carlo FR/RSS method described by P98b. For each Monte Carlo realization, we start with a parent light curve of $N$ data points and from this make $N$ independent random selections of these data points without consideration for previous selections. The redundant selections are then discarded, leaving a new light curve of $M \leq N$ points; typically, the fraction of points from the parent light curve that remain unselected in each realization is $\sim 1 / e$. The RSS method seems to successfully account for uncertainties due to effects of individual data points. Another major source of uncertainty is the uncertainty in the measured continuum and emission-line fluxes, and these can be significant if the amplitude of variability is not much larger than the flux errors in individual data points. We attempt to account for this by altering the fluxes of the $M$ data points in each Monte Carlo realization by random Gaussian deviates scaled by the flux error associated with the data point. The FR process is repeated for many independent realizations (usually 2000 or more for the light curves analyzed here), and for the CCF from each realization, $r_{\max }, \tau_{\text {peak }}$, and $\tau_{\text {cent }}$ are recorded. These are used to build up "cross-correlation peak distributions (CCPDs)" and "cross-correlation centroid distributions (CCCDs)" for $\tau_{\text {peak }}$ and $\tau_{\text {cent }}$, respectively. This is done because the distributions of these values are rarely even approximately Gaussian (Maoz \& Netzer 1989).

Welsh (1999) points out a number of potential problems with cross-correlation methodology, some of which can produce biases in determination of the emission-line lags. However, simulations that mimic as much as possible real AGN behavior have not revealed any strong systematic biases. Nevertheless, we caution that cross-correlation of light curves to measure emission-line lags is a rather crude tool, but one that seems to be effective with the limited AGN variability data available at present. Welsh has also suggested a number of modification to the FR/RSS method, and we have extended the Monte Carlo simulations described by P98b to test these suggestions under what we regard as reasonably realistic conditions, and we describe our results below. 
Flux uncertainties and redundant selections.-Welsh (1999) suggests an alternative strategy to FR/RSS, namely, counting redundant selections in each realization and appropriately reducing the flux error for multiply selected points. Specifically, the flux uncertainty for each point that is selected $1 \leq n \leq N$ times should be reduced by a factor of $n^{1 / 2}$. Thus, rather than omitting redundant points, each of the $N$ selections has some real effect on the outcome of the realization, and the weighting is more consistent with the standard bootstrap method (e.g., Diaconis \& Efron 1983) on which the FR/RSS method is based. We carried out detailed simulations like those described by P98b to determine the efficacy of Welsh's proposed scheme. We compare different error assessments by examining the width of the CCPDs and CCCDs produced by simulations; we presume that the algorithm that yields the narrowest CCPDs and CCCDs (i.e., the highest precision measurements) is the best, as long as the errors are not underestimated. Our simulations confirm that the error estimates using Welsh's method are superior to those of the original FR/RSS algorithm. The uncertainties in $\tau_{\text {peak }}$ are typically lower by $\sim 8 \%$, and the uncertainties in $\tau_{\text {cent }}$ are lower by $\sim 3 \%$. Following the procedures of P $98 \mathrm{~b}$, we have also carried out model-dependent Monte Carlo simulations in which we use a known model for the transfer function in order to verify that Welsh's algorithm does not underestimate the uncertainties. We therefore adopt this improvement in the crosscorrelation analysis employed in this contribution.

Detrending the light curves. - Welsh (1999) also suggests that cross-correlation results are more accurate if the data are first "detrended," i.e., a low-order (usually linear) polynomial is fitted to the light curve and subtracted off prior to carrying out the cross-correlation analysis. Our simulations support this, but only in the case where the sampling is excellent, i.e., long duration at high resolution (for the cases considered by P98b, say, a 200 day experiment with observations once per day). However, we find that under conditions of more marginal sampling (say, a 200 day experiment with only 40 observations), which includes nearly all reverberation data that exist, detrending either makes little difference or can, in fact, lead to occasional gross errors in the lag determination and, consequently, gross overestimates of the uncertainties in the lags. For this reason, we elect to not detrend our data prior to cross-correlation.

Peak or centroid? - Whether or not the cross-correlation lag is better characterized by the peak or centroid of the CCF has been debated on many occasions. The advantage of the centroid is that it is related to the centroid of the transfer function and is better defined when the CCF has a broad peak, and for these reasons, we prefer it. Welsh's simulations, however, suggest that the peak is a more robust measure than the centroid, quite at odds with what was found by P98b and contrary to our general experience. The difference seems to be attributable to differences in the transfer functions used by Welsh and by P98b; P98b used only thin-shell and thick-shell transfer functions, which are flat-topped (i.e., with poorly defined peaks), whereas Welsh used only Gaussian transfer functions. If we repeat the simulations of P98b with Gaussian transfer functions, we find little reason to prefer one measure to the other. Further investigation of this issue using real data, as described in this paper, leads us to continue to prefer the CCF centroid, although the CCD peak is also an acceptable way to characterize the emission-line lags.

Centroid threshold.-Aliasing effects can lead to complex structures in CCFs; rarely is the principal peak in the CCF isolated and well-defined. It is therefore necessary to compute the CCF centroid only over a restricted range, including points with values larger than some fixed fraction of the peak value $r_{\max }$. It is conventional to use a threshold of $0.8 r_{\max }$ for this computation, although in some cases where the peak is noisy, lower thresholds are used. In some cases, the centroid can vary significantly for different threshold selections (Koratkar \& Gaskell 1991), although this appears to be less of a problem with well-sampled data (e.g., Dietrich et al. 1993). Simulations based on those of P98b, however, show that a threshold value of $0.8 r_{\max }$ is generally a good choice. With lower thresholds, we find that the CCCD is significantly broadened. For a threshold of $0.5 r_{\max }$, for example, we find that the width of the CCCD increases by $10 \%-20 \%$, depending somewhat on the details of the transfer function (i.e., more sharply peaked transfer functions give results less sensitive to the selected threshold value, as one might expect). We will therefore continue to use a threshold of $0.8 r_{\max }$, unless otherwise noted.

Alloin, D., Clavel, J., Peterson, B. M., Reichert, G. A., \& Stirpe, G. M. 1994, in Frontiers of Space and Ground-Based Astronomy, ed. W. Wamsteker, M. S. Longair, \& Y. Kondo (Dordrecht: Kluwer), 423

Bevington, P. R. 1969, Data Reduction and Error Analysis for the Physical Sciences (New York: McGraw-Hill), 3

Blandford, R. D., \& McKee, C. F. 1982, ApJ, 255, 419

Boroson, T. A. 2003, ApJ, 585, 647

Boroson, T. A., \& Green, R. F. 1992, ApJS, 80, 109

Cappellari, M., Verolme, E. K, van der Marel, R. P., Verdoes Klejin, G. A., Illingworth, G. D., Franx, M., Carollo, C. M., \& de Zeeuw, P. T. 2002, ApJ, 578,787

Clavel, J., et al. 1991, ApJ, 366, 64

Collier, S., et al. 1998, ApJ, 500, 162

Diaconis, P., \& Efron, B. 1983, Sci. Am., 248, 116

Dietrich, M., et al. 1993, ApJ, 408, 416 1994, A\&A, 284, 33 1998, ApJS, 115, 185

Ferrarese, L., \& Merritt, D. M. 2000, ApJ, 539, L9

Ferrarese, L, Pogge, R. W., Peterson, B. M., Merritt, D., Wandel, A., \& Joseph, C. L. 2001, ApJ, 555, L79

Ford, H. C., et al. 1994, ApJ, 435, L27

Fromerth, M. J., \& Melia, F. 2000, ApJ, 533, 172

Gebhardt, K., et al. 2000a, ApJ, 539, L13 2000b, ApJ, 543, L5

2003, ApJ, 583, 92

\section{EFERENCES}

Harms, R. J., et al. 1994, ApJ, 435, L35

Herrnstein, J. R., et al. 1999, Nature, 400, 539

Ho, L. 1999, in Observational Evidence for Black Holes in the Universe, ed. S. Chakrabarti (Dordrecht: Reidel), 157

Horne, K., Peterson, B. M., Collier, S., \& Netzer, H. 2004, PASP, 116, 465

Jefferys, W. H., Fitzpatrick, M. J., \& McArthur, B. E. 1988, Celest. Mech., 41,39

Kaspi, S., Smith, P. S., Netzer, H., Maoz, D., Jannuzi, B. T., \& Giveon, U. 2000, ApJ, 533, 631

Kaspi, S., et al. 1996, ApJ, 470, 336

Kollatschny, W. 2003, A\&A, 407, 461

Koratkar, A. P., \& Gaskell, C. M. 1991, ApJS, 75, 719

Korista, K. T., et al. 1995, ApJS, 97, 285

Krolik, J. H. 2001, ApJ, 551, 72

Krolik, J. H., Horne, K., Kallman, T. R., Malkan, M. A., Edelson, R. A., \& Kriss, G. A. 1991, ApJ, 371, 541

Macchetto, F., Marconi, A., Axon, D. J., Capetti, A., Sparks, W., \& Crane, P. 1997, ApJ, 489, 579

Maoz, D., \& Netzer, H. 1989, MNRAS, 236, 21

Maoz, D., et al. 1990, ApJ, 351, 75

1991, ApJ, 367, 493

1993, ApJ, 404, 576

Marziani, P., Sulentic, J. W., Zamanov, R., Calvani, J., Dultzin-Hacyan, D.,

Bachev, R., \& Zwitter, T. 2003, ApJS, 145, 199

McLure, R. J., \& Dunlop, J. S. 2002, MNRAS, 331, 795 
McLure, R. J., \& Jarvis, M. J. 2002, MNRAS, 337, 109

Miyoshi, M., Moran, J., Herrnstein, J., Greenhill, L., Nakai, N., Diamond, P., \& Inoue, M. 1995, Nature, 373, 127

Netzer, H. 1990, in Active Galactic Nuclei, ed. T. J.-L. Courvoisier and M. Major (Berlin: Springer), 57 2003, ApJ, 583, L5

Netzer, H., et al. 1990, ApJ, 353, 108

O’Brien, P. T., et al. 1998, ApJ, 509, 163

Onken, C. A., Ferrarese, L., Merritt, D., Peterson, B. M., Pogge, R. W., Vestergaard, M., \& Wandel, A. 2004, ApJ, in press

Onken, C. A., \& Peterson, B. M. 2002, ApJ, 572, 746

Onken, C. A., Peterson, B. M., Dietrich, M., Robinson, A., \& Salamanca, I. M. 2003, ApJ, 585, 121

Osterbrock, D. E., \& Shuder, J. M. 1982, ApJS, 49, 149

Peterson, B. M. 1993, PASP, 105, 247 . 1999, in Structure and Kinematics of Quasar Broad Line Regions, ed. C. M. Gaskell, W. N. Brandt, D. Dultzin-Hacyan, M. Dietrich, \& M. Eracleous (San Francisco: ASP), 49 2001, in Advanced Lectures on the Starburst-AGN Connection, ed. I. Aretxaga, D. Kunth, \& R. Mújica (Singapore: World Scientific), 3

Peterson, B. M., Meyers, K. A., \& Capriotti, E. R. 1984, ApJ, 283, 529

Peterson, B. M., \& Wandel, A. 1999, ApJ, 521, L95 2000, ApJ, 540, L13

Peterson, B. M., Wanders, I., Bertram, R., Hunley, J. F., Pogge, R. W., \& Wagner, R. M. 1998a, ApJ, 501, 82

Peterson, B. M., Wanders, I., Horne, K., Collier, S., Alexander, T., \& Kaspi, S. 1998b, PASP, 110, 660 (P98b)

Peterson, B. M., et al. 1991, ApJ, 368, 119 2000, ApJ, 542, 161 2002, ApJ, 581, 197

Pogge, R. W., \& Martini, P. 2002, ApJ, 569, 624

Reichert, G. A., et al. 1994, ApJ, 425, 582

Reynolds, C. S., \& Nowak, M. A. 2003, Phys. Rep., 377, 389

Robinson, A. 1994, in Reverberation Mapping of the Broad-Line Region of Active Galactic Nuclei, ed. P. M. Gondhalekar, K. Horne, \& B. M. Peterson (San Francisco: ASP), 147
Rodríguez-Pascual, P. M., et al. 1997, ApJS, 110, 9

Romanishin, W., et al. 1995, ApJ, 455, 516

Salamanca, I. M., et al. 1994, A\&A, 282, 742

Santos-Lleó, M., et al. 1997, ApJS, 112, 271 2001, A\&A, 369, 57

Savage, B. D., \& Mathis, J. S. 1979, ARA\&A, 17, 73

Schlegel, D. J., Finkbeiner, D. P., \& Davis, M. 1998, ApJ, 500, 525

Schmidt, M., \& Green, R. F. 1983, ApJ, 269, 352

Shemmer, O., Uttley, P., Netzer, H., McHardy, I. M. 2003, MNRAS, 343, 1341

Stirpe, G. M., et al. 1994, ApJ, 425, 609

Valluri, M., Merritt, D., \& Emsellem, E. 2004, ApJ, 602, 66

van der Marel, R. P. 1994, MNRAS, 270, 271

van der Marel, R. P., Cretton, N., de Zeeuw, P. T., \& Rix, H. 1998, ApJ, 493, 613

van Groningen, E., \& Wanders, I. 1992, PASP, 104, 700

Verdoes Kleijn, G. A., van der Marel, R. P., Carollo, C. M., \& de Zeeuw, P. T. 2000, AJ, 120, 1221

Verolme, E. K, et al. 2002, MNRAS, 335, 517

Véron-Cetty, M. P.,\& Véron, P. 2001, A\&A, 374, 92

Vestergaard, M. 2002, ApJ, 571, 733 . 2004, ApJ, 601, 676

Wandel, A. 2002, ApJ, 565, 762

Wandel, A., Peterson, B. M., \& Malkan, M. A. 1999, ApJ, 526, 579 (Paper I)

Wanders, I., Peterson, B. M., Pogge, R. W., DeRobertis, M. M., van Groningen, E. 1992, A\&A, 266, 72

Wanders, I., et al. 1993, A\&A, 269, 39 . 1997, ApJS, 113, 69

Welsh, W. F. 1999, PASP, 111, 1347

White, R. J., \& Peterson, B. M. 1994, PASP, 106, 879

Whittle, M. 1992, ApJS, 79, 49

Wills, B. J., et al. 1993, ApJ, 410, 534

Winge, C., Peterson, B. M., Horne, K., Pogge, R. W., Pastoriza, M. G., \& Storchi-Bergmann, T. 1995, ApJ, 445, 680

Winge, C., Peterson, B. M., Pastoriza, M.G., Storchi-Bergmann, T. 1996, ApJ, 469,648 